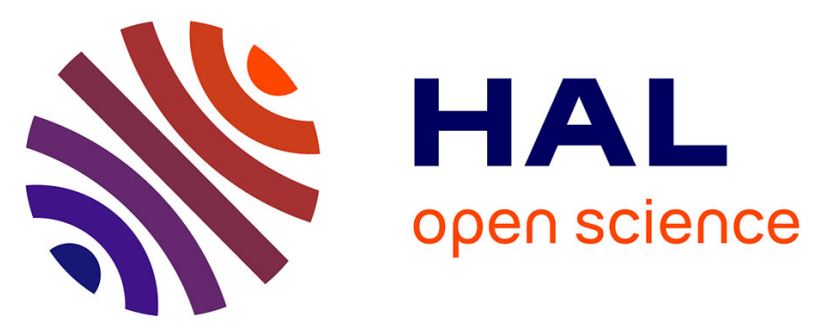

\title{
Radiative transfer within non Beerian porous media with semitransparent and opaque phases in non equilibrium: Application to reflooding of a nuclear reactor
}

\author{
Miloud Chahlafi, Fabien Bellet, Florian Fichot, J. Taine
}

\section{- To cite this version:}

Miloud Chahlafi, Fabien Bellet, Florian Fichot, J. Taine. Radiative transfer within non Beerian porous media with semitransparent and opaque phases in non equilibrium: Application to reflooding of a nuclear reactor. International Journal of Heat and Mass Transfer, 2012, 55 (13-14), pp.3666-3676. 10.1016/j.ijheatmasstransfer.2012.02.067 . hal-00680676

\section{HAL Id: hal-00680676 https://hal.science/hal-00680676}

Submitted on 24 Apr 2012

HAL is a multi-disciplinary open access archive for the deposit and dissemination of scientific research documents, whether they are published or not. The documents may come from teaching and research institutions in France or abroad, or from public or private research centers.
L'archive ouverte pluridisciplinaire HAL, est destinée au dépôt et à la diffusion de documents scientifiques de niveau recherche, publiés ou non, émanant des établissements d'enseignement et de recherche français ou étrangers, des laboratoires publics ou privés. 


\title{
Radiative transfer within non Beerian porous media with semitransparent and opaque phases in non equilibrium; Application to reflooding of a nuclear reactor.
}

\author{
Miloud Chahlafi ${ }^{\mathrm{a}, \mathrm{b}, \mathrm{c}}$, Fabien Bellet ${ }^{\mathrm{a}, \mathrm{b}}$, Florian Fichot ${ }^{\mathrm{c}}$, Jean Taine ${ }^{\mathrm{a}, \mathrm{b}, *}$ \\ ${ }^{a}$ CNRS, UPR 288, Laboratoire d'Énergétique Moléculaire et Macroscopique, Combustion (EM2C), \\ ECP, Bâtiment Péclet, 92295 Châtenay-Malabry, France. \\ ${ }^{b}$ École Centrale Paris, Bâtiment Péclet, F-92295 Châtenay-Malabry, France. \\ ${ }^{c}$ Institut de Radioprotection et de Sûreté Nucléaire, \\ Cadarache BP3, 13115 Saint-Paul-Lez-Durance Cedex, France.
}

\begin{abstract}
A local radiative transfer model is developed for strongly anisotropic porous media with an opaque phase and a mixture of two semitransparent phases. At the optically thick limit, the homogenized phase associated with the opaque interfaces is characterized by generalized extinction and scattering coefficients at equilibrium, a phase function and an effective refraction index, by following the model of Taine et al.[1] for non Beerian media. The radiative transfer model is based on a Radiative Transfer Equation (RTE) with three source terms, which are associated with the temperature fields of the opaque interfaces and the two semitransparent phases. This RTE has been solved by a perturbation technique, which allows radiative interfacial fluxes and radiative powers per unit volume, that are exchanged between phases, to be computed at local scale. The main contributions are obtained at zeroth order perturbation. Corrective contributions at first order perturbation are also determined: Radiative fluxes and powers are then expressed from coupled Fourier's laws, which are characterized by radiative conductivity tensors associated with each phase.

Illustrative results are given for the radiative modeling of reflooding of a damaged
\end{abstract}

\footnotetext{
${ }^{*}$ Corresponding author

Email address: jean.taine@ecp.fr (Jean Taine)
} 
nuclear reactor core. Pragmatic conclusions on the cooling efficiency by steam and water droplets are finally given.

Keywords: Statistical Homogenization, Monte Carlo simulation, Non Beerian Porous Medium, Non Equilibrium, Perturbation Technique.

\section{Nomenclature}

\section{Latin symbols}

$\mathcal{P} \quad$ Radiative power per unit volume

A Specific area per unit volume of the whole porous medium

$B \quad$ Generalized extinction coefficient at equilibrium

E Extinction point

$f_{v} \quad$ Fraction of particles per unit volume

$G$ Cumulative distribution function

$g \quad$ Scattering asymmetry parameter

I Intensity

K Generalized absorption coefficient at equilibrium

$k \quad$ Radiative conductivity tensor

$M \quad$ Current point

$n \quad$ Refractive index

$P \quad$ Cumulative probability

$p \quad$ Scattering phase function

$p_{v} \quad$ Steam pressure 
$q_{i} \quad$ Radiative flux vector (tensorial)

$s \quad$ Curvilinear abscissas along a ray

$T$ Temperature

$u_{i}, u \quad$ Director cosine of axis

$x, y, z$ Coordinate axis

Kn Knudsen number

Greek symbols

$\alpha \quad$ Interface absorptivity

$\kappa \quad$ Absorption coefficient

$\Omega \quad$ Solid angle

$\omega \quad$ Albedo

$\Pi \quad$ Porosity

$\Sigma \quad$ Generalized scattering coefficient at equilibrium

$\sigma \quad$ Scattering coefficient

$\sigma_{s} \quad$ Stefan's constant

$\theta, \varphi \quad$ Euler angles

$\varphi \quad$ Radiative flux

Indexes

$-^{\prime} \quad$ At the calculation point

$-^{+}$Dimensionless 
- $^{(j)} \quad j^{\text {th }}$ order of perturbation

$-_{d} \quad$ Water droplets

$-v \quad$ Water vapor (steam)

$-{ }_{w} \quad$ Homogenized phase $w$ representing the interfaces

$-{ }_{\nu} \quad$ Frequency

$-_{a} \quad$ Absorption

$-_{c} \quad$ Phase $c(w, v$ or $d)$

$-_{\text {ext }}$ Extinction

$-_{e} \quad$ Emission

$-_{i} \quad$ Direction $i$

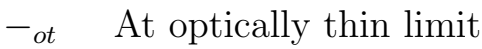

$-_{s c} \quad$ Scattering 


\section{Introduction}

Radiative transfer in porous media is commonly encountered in energy technologies, for instance in ceramic foams for catalytic combustion, in packed beds used in solar absorbers and solar thermochemical reactors, in degraded rod bundles of a nuclear reactor for the modeling of a severe accident, in reformers for $H_{2}$ production, ... For all these applications, an accurate characterization of the radiative properties of the porous medium and the modeling of radiative couplings between different phases are key steps.

The porous medium characterization is generally based on the non always proved assumption that the medium can be modeled by an equivalent semitransparent medium which follows Beer's law. A popular method for characterizing radiative properties consists in identifying a set of parameters that characterize extinction, absorption or scattering from experimental data, for instance reflected or transmitted fluxes [2-7]. A more detailed bibliography is given in Ref.[8]. These approaches are pertinent only if Beer's laws is valid. Because of the large number of parameters to determine, the parameter identification techniques practically always lead to a solution, but do not always allow the obtained set of parameters to be physically validated.

The recent developments of $\gamma$-ray and X-ray tomography techniques allow the morphology of most porous media to be accurately determined. By using morphological data issued from tomographies, Lipinski et al. [9] have used a generalization of the Method of Volume Averaging of Whitaker [10, 11], initiated by Consalvi et al. [12], for characterizing radiative transfer within a porous medium. The specific features of radiation are then only taken into account by an averaged Radiative Transfer Equation (RTE). This approach is limited to a phase which is semitransparent at local scale. As radiation modeling is then degraded by upscaling through a spatial averaging technique, it seems difficult, in this method, to account for coupling at pore scale with other heat transfer modes.

A recent statistical approach leads to a complete characterization of a porous medium by radiative statistical functions. It has been developed by Tancrez and Taine [13] for isotropic and homogeneous porous media. The associated numerical tool is a specific 
Monte Carlo approach. Zeghondy et al. $[14,15]$ have generalized this approach for a statistically anisotropic porous medium, from data issued from tomography and the medium radiative properties at a spatial scale which is smaller than the tomography resolution. Petrasch et al. [16], Haussener et al. [17, 18] have applied the same method to determine the radiative properties of high porosity reticulated ceramics and packed beds used in solar absorbers and solar thermochemical reactors. Bellet et al. [19] have also characterized, by the same approach, bundles of opaque rods of an intact nuclear core, which are strongly anisotropic media. All the previous authors have identified the computed radiative statistical functions with exponential functions, i.e. with functions that follow Beer's law. This approach has been called Radiative Distribution Function Identification (RDFI) in Ref.[14].

In fact, the porous media with an opaque phase and a transparent one which are encountered in Ref. [19] do not follow Beer's law and many other cases of non Beerian media have also been encountered $[1,20]$. A Generalized Radiative Transfer Equation (GRTE), which is directly based on the radiative statistical distribution functions, has been recently introduced for radiative transfer computations in these non Beerian media [1]. By using a perturbation technique of the GRTE, a radiative conductivity tensor has been directly determined by the same authors for non Beerian porous media with opaque and transparent phases. An accurate validity criterion of the associated Fourier's law has also been recently defined [21].

The aim of this paper is to generalize the previous statistical approach to statistically anisotropic porous media with opaque and semitransparent phases and develop an original model of radiative transfer, suitable for non Beerian homogenized phases. Sec.2 deals with this physical model, more precisely with the expressions of the radiative power per unit volume within each semitransparent phase and of the radiative flux at each opaque interface. The radiative energies which are exchanged within an elementary volume $\mathrm{d} V$ of the porous medium and between $\mathrm{d} V$ and its surrounding are then determined. In Sec.3, the method is applied to the radiation model associated with the reflooding of a damaged nuclear core, at the beginning of a severe accident. 


\section{Physical model}

This section deals with the development of an original radiative transfer model for a porous medium with an opaque phase and two semitransparent ones (water droplets and steam, for instance). This medium is statistically homogeneous and possibly anisotropic. A key preliminary step is to characterize a homogenized continuous phase associated with the opaque solid interface elements. In this model, the geometrical optics laws are considered valid because the typical pore size is assumed much larger than the typical radiation wavelength. Diffraction effects are then neglected. The morphology of the opaque walls is characterized by data issued from tomography images. As justified in the following, a first spatial limitation of the radiative properties of the homogenized phase is linked to the spatial resolution of these images. Another limitation comes from the spatial resolution of the temperature field, which is involved in emission and in fact depends on the type of model used for the other heat transfer modes [22]. In the following, the smallest spatial scale which fulfills the two previous conditions is called local scale.

\subsection{Radiative statistical functions}

As detailed in Taine et al. [1, 23], the radiative properties of a statistically anisotropic porous medium with an opaque solid phase and a transparent fluid one are completely characterized by four radiative statistical functions: i) An extinction cumulative distribution function $G_{e x t}\left(s^{\prime}-s, \theta, \varphi\right)$, independent of the radiation frequency $\nu$; it is, in fact, the cumulative distribution function of lengths of all segments $M E$ joining any point $M(s)$ of the fluid phase to any extinction point $E\left(s^{\prime}\right)$ at the fluid-solid interface for a given direction of propagation $\mathbf{u}(\theta, \varphi)$; ii) An absorption (or scattering) cumulative probability from $M$ to $E, P_{a, \nu}\left(s^{\prime}-s, \theta, \varphi\right)$ (or $\left.P_{s c, \nu}\left(s^{\prime}-s, \theta, \varphi\right)\right)$. For a diffuse reflection law at the wall, characterized by an absorptivity $\alpha_{\nu}$, the previous functions are linked by

$$
P_{a, \nu}\left(s^{\prime}-s, \theta, \varphi\right)=1-P_{s c, \nu}\left(s^{\prime}-s, \theta, \varphi\right)=\alpha_{\nu} G_{e x t}\left(s^{\prime}-s, \theta, \varphi\right) .
$$

iii) A scattering phase function $p_{w}\left(\theta_{i}, \varphi_{i}, \theta_{s c}, \varphi_{s c}\right)$, independent of the radiation frequency $\nu$ and depending on both the incidence direction $\left(\theta_{i}, \varphi_{i}\right)$ and the scattering 
one $\left(\theta_{s c}, \varphi_{s c}\right)$; iv) An effective refractive index $n_{w, \nu}(\theta, \varphi)$ depending on direction $(\theta, \varphi)$, if $P_{s c, \nu}\left(s^{\prime}-s, \theta, \varphi\right)$ depends on this direction [19, 1]. The spatial resolution of these functions is only limited by the resolution of the tomography, which defines the porous medium morphology.

The GRTE, developed in Ref.[1, 21] for possibly non Beerian phases of this kind of porous media, is directly based on $G_{e x t}\left(s^{\prime}-s, \theta, \varphi\right), P_{s c, \nu}\left(s^{\prime}-s, \theta, \varphi\right), n_{w, \nu}(\theta, \varphi)$ and the scattering phase function $p_{w}\left(\theta_{i}, \varphi_{i}, \theta_{s c}, \varphi_{s c}\right)$.

For optically thick media, the GRTE rigorously degenerates into a classical RTE, characterized by generalized extinction, absorption and scattering coefficients at equilibrium $B_{w}(\theta, \varphi), K_{w, \nu}(\theta, \varphi)$ and $\Sigma_{w, \nu}(\theta, \varphi)$ given by

$$
\begin{aligned}
B_{w}(\theta, \varphi) & =\left(\int_{0}^{\infty}\left[1-G_{e x t}(\zeta, \theta, \varphi)\right] \mathrm{d} \zeta\right)^{-1}, \\
K_{w, \nu}(\theta, \varphi) & =\alpha_{\nu} B(\theta, \varphi), \\
\Sigma_{w, \nu}(\theta, \varphi) & =B_{w}(\theta, \varphi)-K_{w, \nu}(\theta, \varphi)=\left(1-\alpha_{\nu}\right) B(\theta, \varphi) .
\end{aligned}
$$

More precisely, this RTE is valid [21]: i) In the core of the porous medium, i.e. at a distance from any system boundary larger than 5 to $10 K_{\text {eff }}^{-1}$, where $K_{\text {eff }}(\omega, g)$ is an effective absorption coefficient which accounts for scattering and is a function of the albedo $\omega$ and the scattering asymmetry parameter $g$; ii) When the Gomart and Taine criterion is fulfilled, i.e.

$$
K_{e f f}(\omega, g) \delta>\frac{\mathrm{d} T}{T} \eta
$$

where $\mathrm{d} T$ is the temperature variation along any spatial scale $\delta$ and $\eta$ is a constant parameter which depends on the wished level of accuracy on Fourier's law. The conditions i) and ii) are assumed fulfilled in the following for the spatial scale $\delta$.

\subsection{Radiative transfer equation with three source terms}

Radiative transfer within a porous medium with a solid opaque phase and a semitransparent gas-particle mixture (water droplets and steam) is now considered, at the local scale which has been defined in Sec. 2.1. More precisely, three phases, i.e. a homogenized phase associated with opaque solid interfaces $w$, as developed in Sec. 
2.1 and in Refs. [1] and [19], water vapor $v$ and water droplets $d$, are accounted for. They are characterized by different temperature fields: $T_{w}, T_{v}$ and $T_{d}$, respectively. The specificity of the considered case is that the radiation intensities which are emitted by the three phases are associated with the same propagation medium, i.e. only the fluid phase. The gas absorbs radiation and does not scatter it. The particles absorb radiation and possibly scatter it. The homogenized phase $w$ and the gaseous phase $v$ are characterized by the refractive indices $n_{w, \nu}(\theta, \varphi)$ and $n_{v, \nu}$, which is in practice equal to 1 . At any point, the whole medium is the superimposition of the homogenized phase $w$ and the gas-particle mixture $(v, d)$. Indeed, this mixture is considered as a unique phase whose effective extinction and absorption coefficients are the sum of the extinction and absorption coefficients of the gas and the particles. Particle radiation is treated, as commonly done, under the far field approximation. As the porosity $\Pi$ is the presence probability of the fluid phase within any volume element $\mathrm{d} V$ of the whole porous medium, the homogenized phase $w$ is also characterized by the same porosity within $\mathrm{d} V$ [23]. The conservation laws between the virtual propagation phases $w$ and the real one $v$, i.e. Clausius theorem[24] and flux conservation, are applied to corresponding rays through a fictitious elementary surface $\mathrm{d} S$ belonging to both propagation phases $w$ and $v$. Clausius theorem reads

$$
n_{w, \nu}^{2} \cos \theta_{w} \mathrm{~d} S \mathrm{~d} \Omega_{w}=n_{v, \nu}^{2} \cos \theta_{v} \mathrm{~d} S \mathrm{~d} \Omega_{v}
$$

where $\theta_{w}$ and $\theta_{v}$ are the angles between the unit vector which is normal to $\mathrm{d} S$ and the propagation directions of the rays. Moreover, an incident ray is transmitted in the same direction in the other phase, i.e. $\cos \theta_{w}=\cos \theta_{v}$, and Eq. (6) becomes

$$
n_{w, \nu}^{2} \mathrm{~d} \Omega_{w}=n_{v, \nu}^{2} \mathrm{~d} \Omega_{v}
$$

The flux conservation between $w$ and $v$ reads, at any point of the homogenized volume,

$$
I_{w, \nu} \cos \theta_{w} \mathrm{~d} \Omega_{w}=I_{v, \nu} \cos \theta_{v} \mathrm{~d} \Omega_{v}
$$


where $I_{w, \nu}$ and $I_{v, \nu}$ are the intensities within the propagation phases $w$ and $v$, respectively. Eq. (8) becomes

$$
I_{w, \nu} \mathrm{d} \Omega_{w}=I_{v, \nu} \mathrm{d} \Omega_{v}
$$

Eqs. (7) and (9) prove then the conservation of the intensity under vacuum between $w$ and $v$, which reads: $I_{w, \nu} / n_{w, \nu}^{2}=I_{v, \nu} / n_{v, \nu}^{2}$.

For instance, the spectral absorbed flux is the sum, at any point, of the spectral fluxes absorbed by the phases $w, v$ and $d$, i.e.

$$
\frac{\mathrm{d} \Phi_{\nu}^{a}}{\mathrm{~d} \nu}=K_{w, \nu} I_{w, \nu} \mathrm{d} \Omega_{w}+\left(\kappa_{v, \nu}+\kappa_{d, \nu}\right) I_{v, \nu} \mathrm{d} \Omega_{v} .
$$

With the previous conservation laws given by Eqs. (7) and $(9), \mathrm{d} \Phi_{\nu}^{a} / \mathrm{d} \nu$ can be written either in the propagation phase $w$ or in the propagation phase $v$, i.e.

$$
\frac{\mathrm{d} \Phi_{\nu}^{a}}{\mathrm{~d} \nu}=\left[K_{w, \nu}(\mathbf{u})+\kappa_{v, \nu}+\kappa_{d, \nu}\right] I_{w, \nu} \mathrm{d} \Omega_{w}=\left[K_{w, \nu}(\mathbf{u})+\kappa_{v, \nu}+\kappa_{d, \nu}\right] I_{v, \nu} \mathrm{d} \Omega_{v} .
$$

In the same way, the spectral elementary radiative fluxes which are emitted, $\mathrm{d} \Phi_{\nu}^{e} / \mathrm{d} \nu$, extinguished by scattering, $\mathrm{d} \Phi_{\nu}^{s c^{-}} / \mathrm{d} \nu$, and issued from the scattering source term, $\mathrm{d} \Phi_{\nu}^{s c^{+}} / \mathrm{d} \nu$ are given in the phase $v$ by

$$
\begin{aligned}
\frac{\mathrm{d} \Phi_{\nu}^{e}}{\mathrm{~d} \nu}= & \Pi\left[K_{w, \nu}(\mathbf{u}) I_{\nu}^{\circ}\left(T_{w}\right)+\kappa_{v, \nu} I_{\nu}^{\circ}\left(T_{v}\right)+\kappa_{d, \nu} I_{\nu}^{\circ}\left(T_{d}\right)\right] n_{v, \nu}^{2} \mathrm{~d} \Omega_{v} \\
\frac{\mathrm{d} \Phi_{\nu}^{s c^{-}}}{\mathrm{d} \nu}= & {\left[\Sigma_{w, \nu}(\mathbf{u})+\sigma_{d, \nu}\right] I_{v, \nu} \mathrm{d} \Omega_{v} } \\
\frac{\mathrm{d} \Phi_{\nu}^{s c^{+}}}{\mathrm{d} \nu}= & {\left[\frac{1}{n_{w, \nu}^{2}(\mathbf{u})} \int_{4 \pi} \Sigma_{w, \nu}\left(\mathbf{u}^{\prime}\right) p_{w}\left(\mathbf{u}^{\prime}, \mathbf{u}\right) n_{w, \nu}^{2}\left(\mathbf{u}^{\prime}\right) I_{v, \nu}\left(\mathbf{u}^{\prime}\right) \mathrm{d} \Omega^{\prime}\right.} \\
& \left.+\sigma_{d, \nu} \int_{4 \pi} p_{d, \nu}\left(\mathbf{u}^{\prime} \cdot \mathbf{u}\right) I_{v, \nu}\left(\mathbf{u}^{\prime}\right) \mathrm{d} \Omega^{\prime}\right] \frac{\mathrm{d} \Omega_{v}}{4 \pi}
\end{aligned}
$$

where $\sigma_{d, \nu}$ and $p_{d, \nu}\left(\mathbf{u}^{\prime} \cdot \mathbf{u}\right)$ are the scattering coefficient of the particles and their scattering phase function, which only depends on the scattering angle cosine $\mathbf{u}^{\prime} \cdot \mathbf{u}$. 
The RTE simply reads

$$
\frac{\mathrm{d} I_{w, \nu}}{\mathrm{d} s} \mathrm{~d} \Omega_{w}=\frac{\mathrm{d} I_{v, \nu}}{\mathrm{d} s} \mathrm{~d} \Omega_{v+d}=-\frac{\mathrm{d} \Phi_{\nu}^{a}}{\mathrm{~d} \nu}-\frac{\mathrm{d} \Phi_{\nu}^{s c^{-}}}{\mathrm{d} \nu}+\frac{\mathrm{d} \Phi_{\nu}^{e}}{\mathrm{~d} \nu}+\frac{\mathrm{d} \Phi_{\nu}^{s c^{+}}}{\mathrm{d} \nu} .
$$

It can also be expressed in phase $v$, i.e.

$$
\begin{aligned}
\frac{\mathrm{d} I_{v, \nu}}{\mathrm{d} s}= & -\left[B_{w}(\mathbf{u})+\kappa_{v, \nu}+\kappa_{d, \nu}+\sigma_{d, \nu}\right] I_{v, \nu} \\
& +\Pi n_{v, \nu}^{2}\left[K_{w, \nu}(\mathbf{u}) I_{\nu}^{\circ}\left(T_{w}\right)+\kappa_{v, \nu} I_{\nu}^{\circ}\left(T_{v}\right)+\kappa_{d, \nu} I_{\nu}^{\circ}\left(T_{d}\right)\right] \\
& +\frac{\Sigma_{w, \nu}(\mathbf{u})}{4 \pi} \int_{4 \pi} p_{w}\left(\mathbf{u}^{\prime}, \mathbf{u}\right) I_{v, \nu}\left(\mathbf{u}^{\prime}\right) \mathrm{d} \Omega^{\prime} \\
& +\frac{\sigma_{d, \nu}}{4 \pi} \int_{4 \pi} p_{d, \nu}\left(\mathbf{u}^{\prime} . \mathbf{u}\right) I_{v, \nu}\left(\mathbf{u}^{\prime}\right) \mathrm{d} \Omega^{\prime}
\end{aligned}
$$

where the fact that the product $n_{w, \nu}^{2} \Sigma_{w, \nu}$ is constant [19] has been used. The RTE given by Eq. (16) is solved by a perturbation technique in order to obtain expressions, at local scale, of the radiative power per unit volume within any phase and the radiative flux at the opaque interface. After introduction of the dimensionless abscissa $s^{+}=s / \delta$ in Eq. (16) and division of all its terms by $n_{w, \nu}^{2} B_{w}+\kappa_{v, \nu}+\kappa_{d, \nu}+\sigma_{d, \nu}$, the transport term of the RTE becomes $\mathrm{Kn}^{R} \frac{\mathrm{d} I_{v, \nu}}{\mathrm{d} s^{+}}$, in which appears the radiative Knudsen's number $\mathrm{Kn}^{R}$, perturbation parameter which is small compared to 1, i.e.

$$
\mathrm{Kn}^{R}=\frac{1}{\left(n_{w, \nu}^{2} B_{w}+\kappa_{v, \nu}+\kappa_{d, \nu}+\sigma_{d, \nu}\right) \delta} \ll 1 .
$$

The perturbation solution of Eq. (16) writes: $I_{v, \nu}=I_{v, \nu}^{(0)}+I_{v, \nu}^{(1)}$. It corresponds to a truncated Taylor series with respect to $\mathrm{Kn}^{R} . I_{v, \nu}^{(0)}$ and $I_{v, \nu}^{(1)}$ are the zeroth order and first order solutions of the RTE and are respectively independent of $\mathrm{Kn}^{R}$ and proportional to $\mathrm{Kn}^{R}$. A similar approach is detailed in Refs. [19, 1, 25].

The zeroth order solution $I_{v, \nu}^{(0)}$ allows the radiative powers that are exchanged between the phases within the elementary volume $\mathrm{d} V$ itself to be calculated, as detailed in Sec.2.3. It is the main contribution. The first order corrective solution $I_{v, \nu}^{(1)}$ depends on the transport term, i.e. the first term of the first member of Eq. (16), and allows radiative exchanges between an elementary volume and its environment to be 
determined, as detailed in Sec.2.4.

\subsection{Coupled radiative powers}

After identification of the terms of zeroth order in $\mathrm{Kn}^{R}$ in Eq. (16), the intensity $I_{v, \nu}^{(0)}(\mathbf{u})$ is obtained from the implicit equation

$$
I_{v, \nu}^{(0)}(\mathbf{u})=\prod n_{v, \nu}^{2} \frac{\left[K_{w, \nu}(\mathbf{u}) I_{\nu}^{\circ}\left(T_{w}\right)+\kappa_{v, \nu} I_{\nu}^{\circ}\left(T_{v}\right)+\kappa_{d, \nu} I_{\nu}^{\circ}\left(T_{d}\right)\right]}{B_{w}(\mathbf{u})+\kappa_{v, \nu}+\kappa_{d, \nu}+\sigma_{d, \nu}}+\mathcal{L}_{\nu}\left[\mathbf{u}^{\prime} \mapsto I_{v, \nu}^{(0)}\left(\mathbf{u}^{\prime}\right)\right](\mathbf{u}),
$$

where $\mathcal{L}_{\nu}$ is a dimensionless linear functional defined by

$$
\begin{aligned}
& \mathcal{L}_{\nu}\left[\mathbf{u}^{\prime} \mapsto X\left(\mathbf{u}^{\prime}\right)\right](\mathbf{u}) \\
= & \frac{1}{4 \pi\left[B_{w}(\mathbf{u})+\kappa_{v, \nu}+\kappa_{d, \nu}+\sigma_{d, \nu}\right]} \int_{4 \pi}\left[\Sigma_{w, \nu}(\mathbf{u}) p_{w}\left(\mathbf{u}^{\prime}, \mathbf{u}\right)+\sigma_{d, \nu} p_{d, \nu}\left(\mathbf{u}^{\prime} \cdot \mathbf{u}\right)\right] X\left(\mathbf{u}^{\prime}\right) \mathrm{d} \Omega^{\prime} .
\end{aligned}
$$

The solution of Eq. (18) is the sum of three contributions, associated with emission by the the different phases $w, v$ and $d$, i.e.

$$
I_{v, \nu}^{(0)}(\mathbf{u})=I_{v, \nu}^{w^{(0)}}(\mathbf{u})+I_{v, \nu}^{v^{(0)}}(\mathbf{u})+I_{v, \nu}^{d^{(0)}}(\mathbf{u}),
$$

where for any phase $c(w, v$ or $d) I_{v, \nu}^{c^{(0)}}$ is given by

$$
I_{v, \nu}^{c^{(0)}}(\mathbf{u})=\Pi n_{v, \nu}^{2} I_{\nu}^{\circ}\left(T_{c}\right) \sum_{k=0}^{\infty} \mathcal{L}_{\nu}^{k}\left[\mathbf{u}^{\prime} \mapsto \frac{\kappa_{c, \nu}}{B_{w}\left(\mathbf{u}^{\prime}\right)+\kappa_{v, \nu}+\kappa_{d, \nu}+\sigma_{d, \nu}}\right](\mathbf{u}) .
$$

In Eq. $(21), \mathcal{L}_{\nu}^{k}$ represents the functional $\mathcal{L}_{\nu}$ applied $k$ times and $\kappa_{c, \nu}$ stands for $K_{w, \nu}(\mathbf{u}), \kappa_{v, \nu}$ or $\kappa_{d, \nu}$. The uniform zeroth order radiative power per unit volume associated with a phase $c(w, v$ or $d)$ is then the difference between the emission and absorption contributions, i.e.

$$
\mathcal{P}_{c}^{(0)}=\int_{0}^{\infty} \int_{4 \pi} \kappa_{c, \nu}\left[I_{v, \nu}^{(0)}-\Pi n_{v}^{2} I_{\nu}^{\circ}\left(T_{c}\right)\right] \mathrm{d} \Omega \mathrm{d} \nu
$$

$\mathcal{P}_{c}^{(0)}(c=w, v$ or $d)$ is a source term for the energy balance equation related to the phase $c$ at homogenization scale. Radiation emitted by a phase in an elementary volume $\mathrm{d} V$ at zeroth order is absorbed in the same $\mathrm{d} V$, and the global power $\mathcal{P}_{w}^{(0)}+$ 
$\mathcal{P}_{v}^{(0)}+\mathcal{P}_{d}^{(0)}$ is therefore equal to 0 . A uniform zeroth order radiative flux $\varphi_{w}^{(0)}$, at local interfaces of the considered elementary volume $\mathrm{d} V$, is defined by

$$
\varphi_{w}^{(0)}=\frac{\mathcal{P}_{w}^{(0)}}{A}
$$

where $A$ is the specific area per unit volume of the whole porous medium.

\subsection{Coupled radiative conductivity tensors}

Similarly, the first order solution of Eq. (16) is given by

$$
I_{v, \nu}^{(1)}(\mathbf{u})=-\left(\frac{1}{B_{w}(\mathbf{u})+\kappa_{v, \nu}+\kappa_{d, \nu}+\sigma_{d, \nu}}\right) \frac{\mathrm{d} I_{v, \nu}^{(0)}}{\mathrm{d} s}(\mathbf{u})+\mathcal{L}_{\nu}\left[\mathbf{u}^{\prime} \mapsto I_{v, \nu}^{(1)}\left(\mathbf{u}^{\prime}\right)\right](\mathbf{u}) .
$$

The solution of Eq. (24) is also the sum of the emission contributions of the three phases

$$
I_{v, \nu}^{(1)}(\mathbf{u})=I_{v, \nu}^{w^{(1)}}(\mathbf{u})+I_{v, \nu}^{v^{(1)}}(\mathbf{u})+I_{v, \nu}^{d^{(1)}}(\mathbf{u}),
$$

where, for a phase $c(w, v$ or $d)$,

$$
I_{v, \nu}^{c^{(1)}}(\mathbf{u})=-\sum_{k=0}^{\infty} \mathcal{L}_{\nu}^{k}\left[\mathbf{u}^{\prime} \mapsto\left(\frac{1}{B_{w}\left(\mathbf{u}^{\prime}\right)+\kappa_{v, \nu}+\kappa_{d, \nu}+\sigma_{d, \nu}}\right) \frac{\mathrm{d} I_{v, \nu}^{c^{(0)}}}{\mathrm{d} s}\left(\mathbf{u}^{\prime}\right)\right](\mathbf{u}) .
$$

The zeroth order solution of Eq. (16) does not generate any flux because of symmetries, whereas the first order flux can be expressed as a coupled conductive Fourier's law given, in tensorial notations, by

$$
q_{i}^{(1)}=\int_{0}^{\infty} \int_{4 \pi} I_{v, \nu}^{(1)}(\mathbf{u}) u_{i} \mathrm{~d} \Omega \mathrm{d} \nu=-k_{i j_{w}} \frac{\partial T_{w}}{\partial x_{j}}-k_{i j_{v}} \frac{\partial T_{v}}{\partial x_{j}}-k_{i j_{d}} \frac{\partial T_{d}}{\partial x_{j}}
$$

where the coupled conductivity tensor associated with $c(w, v$ or $d)$ is

$$
k_{i j_{c}}=\int_{0}^{\infty} \int_{4 \pi} \sum_{k=0}^{\infty} \mathcal{L}_{\nu}^{k}\left[\mathbf{u}^{\prime} \mapsto \frac{u_{j}^{\prime}}{B_{w}\left(\mathbf{u}^{\prime}\right)+\kappa_{v, \nu}+\kappa_{d, \nu}+\sigma_{d, \nu}} \frac{\mathrm{d} I_{v, \nu}^{(0)}}{\mathrm{d} T_{c}}\left(\mathbf{u}^{\prime}\right)\right](\mathbf{u}) u_{i} \mathrm{~d} \Omega \mathrm{d} \nu .
$$


In Eq. (27), a term $-k_{i j_{c}} \frac{\partial T_{c}}{\partial x_{j}}$ physically corresponds to fluxes emitted by the phase $c$ at temperature $T_{c}$ and absorbed by the three phases $w, v$ or $d$. The term results in fact from reciprocal exchanges between a volume element and the surrounding elements. The fraction of this term corresponding to emission by a phase $c(w, v$ or $d)$ and absorption by a phase $c^{\prime}(w, v$ or $d)$ is equal to $-F_{c c^{\prime}} k_{i j_{c}} \frac{\partial T_{c}}{\partial x_{j}}$, where $F_{c c^{\prime}}$ is given by

$$
F_{c c^{\prime}}=\frac{\int_{0}^{\infty} \int_{4 \pi} \kappa_{c^{\prime}, \nu} I_{v, \nu}^{c^{(1)}} \mathrm{d} \Omega \mathrm{d} \nu}{\int_{0}^{\infty} \int_{4 \pi}\left[K_{w, \nu}(\mathbf{u})+\kappa_{v, \nu}+\kappa_{d, \nu}\right] I_{v, \nu}^{c^{(1)}} \mathrm{d} \Omega \mathrm{d} \nu} .
$$

Consequently, the radiative power per unit volume emitted by $c$ and absorbed by $c^{\prime}$ is, at the first order of perturbation,

$$
\mathcal{P}_{c c^{\prime}}^{(1)}=\frac{\partial}{\partial x_{i}}\left[F_{c c^{\prime}} k_{i j_{c}} \frac{\partial T_{c}}{\partial x_{j}}\right]
$$

and the radiative power associated with a phase $c$ becomes

$$
\mathcal{P}_{c}^{(1)}=\frac{\partial}{\partial x_{i}}\left[F_{c^{\prime} c} k_{i j_{c^{\prime}}} \frac{\partial T_{c^{\prime}}}{\partial x_{j}}+F_{c^{\prime \prime} c} k_{i j_{c^{\prime \prime}}} \frac{\partial T_{c^{\prime \prime}}}{\partial x_{j}}-\left(F_{c c^{\prime}}+F_{c c^{\prime \prime}}\right) k_{i j_{c}} \frac{\partial T_{c}}{\partial x_{j}}\right] .
$$

This expression is physically meaningful for steam and water droplets $(v$ and $d)$. In the case of wall, the interfacial flux, which is applied at the first order of perturbation is

$$
\varphi_{w}^{(1)}=\frac{\mathcal{P}_{w}^{(1)}}{A} .
$$

It is worth noticing that in most cases the radiative conductivity tensors $k_{i j_{c}}$ are diagonal. Finally Eqs. (22), (23), (31) and (32) define all the radiative source terms of a transfer model which would be applied to the three phases of the material system.

\section{Application to the reflooding of nuclear rod bundles}

The considered porous medium is a small-scale experimental facility of the international program PHEBUS [26] which simulates the degradation process during a 
severe accident of a nuclear reactor. It is a bundle of 21 fuel rods. All the rods of 1 $m$ long and $9.6 \mathrm{~mm}$ diameter are regularly spaced in squared pitch of $1.27 \mathrm{~cm}$. The treatment of data, which are issued from $\gamma$-tomography, is first presented. The determination of the extinction cumulative distribution function $G_{e x t}$ and the scattering phase function $p$ for this experimental system is then detailed. The results related to $G_{\text {ext }}$ and the obtained values of the directional generalized extinction coefficients at equilibrium and directional scattering asymmetry parameters are discussed. The radiative properties of the reflooding fluid, i.e. steam and water droplets, are also characterized. Finally, some results related to the radiative powers per unit volume associated with each phase and to the interfacial flux are discussed.

\subsection{Characterization of the solid phase morphology}

The present work is based on the experimental results of the FPT1 and FPT2 tests, which reproduce two sequences of a severe accident. The rod bundle (Fig.1) has been placed at the center of an experimental reactor core. A first treatment of $\gamma$-tomography data leads to 408 cross-sectional images, equally spaced by $3 \mathrm{~mm}$ from the bottom of the system $(z=-72 \mathrm{~mm})$ to its top $(z=1149 \mathrm{~mm})$ for each test. Each image, as shown in Fig.2, is a $512 \times 512$ medium density matrix of spatial resolution $0.24 \times 0.24 \mathrm{~mm}^{2}$. A numerical process has allowed a $3 \mathrm{D}$-matrix to be generated by interpolation of densities along the vertical $z$-axis.

The porosity $\Pi$ and the specific area per unit volume of the whole medium $A$ have been calculated for all cross-sectional images of FPT1 and FPT2. Study zones i, i.e. set of images such as $\Pi_{i}$ and $A_{i}$ are uniform with an accuracy better than $10 \%$, have been identified: 12 axial zones for FPT1 and 23 zones for FPT2. Examples are given in Fig.3.

The fluid-solid interfaces have been located within these matrix by using the marching cube algorithm, which has been developed by Delesse et al. [27] and applied by Zeghondy et al. to mullite foams [14]. The density threshold, which defines the interface location, is a key parameter. It has been adjusted by equalizing the values of the generalized extinction coefficients defined in Eq. (2) which are obtained from: i) The experimental tomography of an intact small-scale facility; ii) An analytical 
definition of the same geometry [19].

[Figure 1 about here.]

[Figure 2 about here.]

[Figure 3 about here.]

\subsection{Radiative characterization of the opaque interfaces}

The extinction cumulative distribution function $G_{e x t}\left(s^{\prime}-s, \theta, \varphi\right)$ and the scattering phase function $p\left(\theta_{i}, \varphi_{i}, \theta_{s c}, \varphi_{s c}\right)$ have been calculated for the 35 identified zones of FPT1 and FPT2 tests. For each zone $i$, the computational domain is the fluid region which is included in a parallelepipedic volume: i) Defined, as shown in Fig.2, by nine central intact rods; ii) Bounded by two cross-sections $z_{\min }$ and $z_{\max }$. A huge number of rays, typically $10^{10}$ rays, have been generated from randomly chosen points $M$ within the fluid phase, which is assumed transparent at this step, into randomly chosen solid angles; each of them is characterized by a direction $(\theta, \varphi) ; \theta$ is the angle of a ray with the $z$-axis, $\varphi$ the azimuth. A perfect specular reflection law has been applied to each ray which impacts any boundary of the parallelepiped which encompasses the computational domain. This algorithm allows the geometry to be indefinitely extended according to the symmetries of the intact system. Indeed, due to the small size of the domain, a large number of rays would never impact a wall element without this extension. This difficulty is not encountered within a real nuclear reactor core which is much larger than the experimental facility. The ratio of the total number of points $M$ which are generated within the fluid phase to the total number of points which are generated within the entire computational domain statistically converges towards $\Pi$. $A$ is calculated using the marching cube algorithm [27].

Each zone of the small-scale experimental facility was, before its degradation, characterized by some symmetries due to the square arrangement of rods, as shown in Fig.2: i) A perfect mirror symmetry with respect to $\varphi=\pi / 4$; ii) A $\pi / 2$ periodicity in $\varphi$; iii) A perfect mirror symmetry with respect to the cross plane $\theta=\pi / 2$. Finally, a $1 / 8$ 
symmetry in the cross section and $1 / 2$ symmetry in the axial direction are obtained. A given realization of the degraded medium does not exhibit these symmetries for uncontrolled experimental reasons. But, it is assumed that the radiative properties of the degraded rods statistically verify these symmetries, as the effects of gravity can be neglected for small sized zones along $z$-axis. Consequently, the calculations of $G_{\text {ext }}\left(s^{\prime}-s, \theta, \varphi\right)$ and $p\left(\theta_{i}, \varphi_{i}, \theta_{s c}, \varphi_{s c}\right)$ have been carried out for 120 values of $\varphi$ regularly spaced in the range $[0,2 \pi]$ and 17 values of $\cos \theta$ regularly spaced in the range $[-1 ; 1]$, i.e. by shooting rays in $4 \pi$ steradians. Because of the symmetries previously discussed, the radiative statistical functions have been cumulated by folding back the results related to any direction into the corresponding direction which is defined by values of $\theta$ and $\varphi$ in the ranges $[0, \pi / 2]$ and $[0, \pi / 4]$, respectively.

The convergence of the Monte-Carlo method is characterized by a relative standard deviation on $G_{\text {ext }}$ of about $10^{-5}$ between 10 sets of $2 \times 10^{9}$ rays. The ratio of the standard deviation on the scattering phase function to its maximum value is of about $5.10^{-3}$ between 10 sets of $2 \times 10^{9}$ rays. An important result is that the functions $G_{\text {ext }}$ which are associated with a damaged core are not exponential, and consequently do not follow Beer's law, as shown in Fig.4. Moreover, $G_{e x t}(\theta, \varphi)$ is strongly anisotropic. Finally, more than $95 \%$ of rays which are shot in all the directions are extinguished in the range $\left[0,10 / \beta_{\text {ref }}\right]$, in practice $[0,5 \mathrm{~cm}]$. The reference extinction coefficient $\beta_{\text {ref }}$ has been chosen equal to $A /(\pi \Pi)$, as in the case of an intact rod bundle [19]. It appears in Fig.4 that $\ln \left[1-G_{e x t}\left(s^{\prime}-s\right)\right]$ can be approximated by a straight line of slope equal to -1 for small values of $\beta_{r e f}\left(s^{\prime}-s\right)$ and for all directions. Consequently, $G_{\text {ext }}$ verify Beer's law at the optically thin limit with an effective extinction coefficient equal to $\beta_{\text {ref }}=A /(\pi \Pi)$ and the specific area $A$ can also be pragmatically determined from the slope of $G_{\text {ext }}$ at $s^{\prime}-s=0$.

On the other side, $G_{e x t}$ converges at the optically thick limit to an exponential function for all directions. When the two conditions given at the end of Sec.2.1 are fulfilled, a directional generalized extinction coefficient at equilibrium $B(\theta, \varphi)$ is computed from Eq. (2); results are given in Fig.5. Nevertheless, $B(\theta, \varphi)$ has no physical meaning for optical thickness in the range [0.1,3] as discussed in Ref.[1].

The directional scattering asymmetry parameter $g(\theta, \varphi)$ is always negative, as shown 
in Fig.6, and corresponds to a backscattering phenomenon. Consequently, the scattering source term acts as a complementary radiative extinction which will contribute to decrease the radiative conductivities, as developed in Sec.3.4.

[Figure 4 about here.]

[Figure 5 about here.]

[Figure 6 about here.]

\subsection{Radiative properties of steam, water droplets and opaque interfaces}

Radiative properties of steam have been characterized by using a CK model for pressures $p_{v}$ and temperatures $T_{v}$ in ranges $[1,10 \mathrm{~atm}]$ and $\left[T_{\text {sat }}\left(p_{v}\right), 2900 \mathrm{~K}\right]$ respectively, where $T_{\text {sat }}$ is the saturation temperature. These data correspond to typical conditions of a some severe accidents of a nuclear reactor. More precisely, $k$-distribution functions have been calculated from Laplace transform of transmissivities computed from a Statistical Narrow-Band (SNB) model [28], based on a Malkmus formulation [29]. The SNB parameters are issued from Ref.[30]. 43 spectral bands in the range $\left[137.5 \mathrm{~cm}^{-1}, 9312.5 \mathrm{~cm}^{-1}\right]$ are considered. The CK model is based on a 7 points Gauss-Lobato quadrature detailed in Refs. [31, 30].

Radiative properties of spherical water droplets have been computed from Mie theory. The data related to the spectral complex index of liquid water are issued from Ref.[32]. The spherical droplets of radii in the range [25 $\mu \mathrm{m}, 1 \mathrm{~mm}]$ are characterized by a volume fraction $f_{v}$ of $10^{-3}$, corresponding to independent scattering conditions; the effects of droplets are then additive. Moreover, it has been proved that forward scattering by droplets can be neglected for wall temperatures higher than $600 \mathrm{~K}$; No difference with transmission of radiation appears in these conditions. Indeed, the width at half maximum of the phase function is always smaller than the size of the elementary solid angle used for Monte Carlo calculations, excepted for frequencies in the range $\left[137.5 \mathrm{~cm}^{-1}, 387.5 \mathrm{~cm}^{-1}\right]$ in the case of droplets of radius $25 \mu \mathrm{m}$. These frequencies play a non negligible role in the radiative transfer for temperatures smaller than $600 \mathrm{~K}$, of weak interest in sequences of a nuclear severe accident. Consequently 
only emission and absorption by isothermal droplets, of fraction per unit volume equal to $10^{-3}$, are considered in the following.

The solid wall is assumed made of Zirconia $\mathrm{ZrO}_{2}$; its absorptivity is given by Ref. [33] and is shown in Fig.8.

Figure 7 shows that the transmissivities associated with steam, water droplets and opaque interfaces, separately considered, have similar orders of magnitude, in the studied conditions. These three phenomena are then considered as coupled in the following.

[Figure 7 about here.]

\subsection{Results related to radiative powers and fluxes}

Radiative powers per unit volume $\mathcal{P}_{c}^{(0)}$ and $\mathcal{P}_{c}^{(1)}(c=w, v$ or $d)$ respectively given by Eqs. (22) and (31) and radiative conductivities $k_{i j_{c}}$ given by Eq. (28) have been computed for the 35 previously identified zones of FPT1 and FPT2 tests by considering four different fluid phases: i) A transparent one; ii) Steam; iii) Water droplets; iv) A mixture of steam and droplets. More precisely, the zeroth order radiative interfacial flux $\varphi_{w}^{(0)}$ has been determined for the three last cases from the zeroth order solution $I_{v}^{(0)}$ of the GRTE, by using Eq. (23). Similarly, as detailed in Sec.2.4, the radiative conductivity tensor $k_{i j_{c}}$ associated with a phase $c$ can be computed from the first order solution $I_{v}^{(1)}$, as defined by Eq. (28). Due to symmetries discussed in Sec.3.2, it has been analytically shown that the tensor $k_{i j_{c}}$ is diagonal, i.e. $k_{i j_{c}}=0$ for $i \neq j$ and that: $k_{x x_{c}}=k_{y y_{c}} \hat{=} k_{r_{c}} ; k_{z z_{c}}$ is simply noted $k_{z_{c}}$ in the following. As previously discussed, water droplets are assumed isothermal at temperature $T_{s a t}\left(p_{v}\right)$, of fraction per unit volume $f_{v}$ equal to $10^{-3}$ and purely absorbing.

The quantity $F_{c c^{\prime}} k_{i j_{c}}$ corresponds to emission by a phase $c$ and absorption by a phase $c^{\prime}$. The fractions $F_{c c^{\prime}}$ are given by Eq. (29) and have been computed by assuming that the radial gradients of $T_{w}$ and $T_{v}$ are negligible in front of the corresponding axial temperature gradients, as observed in sequences of a severe accident of a nuclear 
core, i.e.

$$
\begin{aligned}
& \frac{\partial T_{c}}{\partial x}<<\frac{\partial T_{c}}{\partial z} \quad \text { and } \quad \frac{\partial T_{c}}{\partial y}<<\frac{\partial T_{c}}{\partial z} \quad(c=w \text { or } v) \text {, } \\
& \frac{\partial T_{d}}{\partial x}=\frac{\partial T_{d}}{\partial y}=\frac{\partial T_{d}}{\partial z}=0
\end{aligned}
$$

Moreover, it has been assumed for estimating the different contributions in this Section, that: $\frac{\partial T_{w}}{\partial z} \simeq \frac{\partial T_{v}}{\partial z}$, which allows the fractions $F_{c c^{\prime}}$ to be explicitly computed from Eq. (29) where

$$
I_{v, \nu}^{c^{(1)}}=-\sum_{k=0}^{\infty} \mathcal{L}_{\nu}^{k}\left[\mathbf{u}^{\prime} \mapsto \frac{u_{z}}{B_{w}\left(\mathbf{u}^{\prime}\right)+\kappa_{v, \nu}+\kappa_{d, \nu}}\left(\frac{\partial I_{v, \nu}^{c^{(0)}}}{\partial T_{w}}\left(\mathbf{u}^{\prime}\right)+\frac{\partial I_{v, \nu}^{c^{(0)}}}{\partial T_{v}}\left(\mathbf{u}^{\prime}\right)\right)\right](\mathbf{u}) \frac{\partial T_{w}}{\partial z} .
$$

Studied cases and associated computed quantities are summarized in Tab.1.

[Table 1 about here.]

A transparent fluid phase is defined as reference case. The associated radiative conductivity $k^{\text {trans }}$ only corresponds to emission and absorption by interfaces.

[Figure 8 about here.]

$k^{\text {trans }}$ has been fitted with a relative error smaller than 0.20, as shown in Fig.9, by simple functions of $\Pi, A$ and $T_{w}$, i.e.

$$
\begin{aligned}
& k_{r}^{\text {trans }}(\mathbf{x})=5.68 \frac{\Pi^{2}}{A} 4 \sigma_{s} T_{w}^{3}(\mathbf{x}) \\
& k_{z}^{\text {trans }}(\mathbf{x})=15.25 \frac{\Pi^{2}}{A} 4 \sigma_{s} T_{w}^{3}(\mathbf{x}) .
\end{aligned}
$$

[Figure 9 about here.]

Exchanges between different phases at zeroth order correspond to exchanges between the solid phase and the semitransparent ones within the same elementary volume of the system. $\mathcal{P}_{w}^{(0)}, \mathcal{P}_{v}^{(0)}$ and $\mathcal{P}_{d}^{(0)}$ are respectively shown in Figs.10-12 for temperatures $T_{w}=2000 \mathrm{~K}$ and $T_{v}=600 \mathrm{~K}$. This choice corresponds to a classical case observed 
in the sequence of a severe accident of a nuclear reactor.

As shown by comparison of the top and bottom graphs of Figs.10 and 11, steam is more efficient at high pressure for cooling hot fuel rods than at atmospheric pressure. Moreover, the comparison of orders of magnitude of $\mathcal{P}_{v}^{(0)}$ and $\mathcal{P}_{d}^{(0)}$ given respectively in Figs.11 and 12 shows that steam at high pressure is only as efficient, for cooling fuel rods, as large water droplets of radius $R_{d}=300 \mu \mathrm{m}$. But these large droplets are much less efficient coolers than small droplets of radius $R_{d}=50 \mu \mathrm{m}$ and same volume fraction $f_{v}=10^{-3}$ (before their complete vaporization). Steam at atmospheric pressure is then a bad cooler. However, it is used in practice, since the pressure has to be decreased in order to prevent the risk of pipe explosion.

[Figure 10 about here.]

[Figure 11 about here.]

[Figure 12 about here.]

Exchanges between adjacent volume elements of the system only appear at first order perturbation. The dimensionless quantities $F_{w w} k_{r_{w}} / k_{r}^{\text {trans }}$ and $F_{w w} k_{z_{w}} / k_{z}^{\text {trans }}$ compare respectively radial and axial radiative conductivities associated with the phase $w$, with and without a semitransparent fluid phase. As shown in Fig.13 (a), the semitransparent fluid phase decreases radial conductivities of about $20 \%$ and, as shown in Fig.13 (b), axial conductivities of about 50\%. These effects can be explained by the following argument. The radiative conductivity associated with a statistically isotropic non Beerian porous medium is simply given [1] by

$$
k^{R}\left(T_{w}\right)=\frac{4 \pi \Pi}{3} \int_{0}^{\infty} \frac{n_{\nu}^{2}}{K_{\nu}+\left(1-g_{\nu}\right) \Sigma_{\nu}} \frac{\mathrm{d} I_{\nu}^{\circ}}{\mathrm{d} T_{w}}\left(T_{w}\right) \mathrm{d} \nu .
$$

In this simpler case, the radiative conductivity is a decreasing function of the absorption coefficient $K_{\nu}$. In the present case, the presence of a semitransparent fluid phase increases the absorption coefficient; consequently, radiative conductivities with a semitransparent fluid phase are smaller than radiative conductivities with a transparent one, as for a statistically isotropic medium ruled by Eq. (38). 
[Figure 13 about here.]

The fractions $F_{c c^{\prime}}$,relative to emission by a phase $c(w, v$ or $d)$ and absorption by a phase $c^{\prime}(w, v$ or $d)$, have been computed from Eqs. (29-33,34). The results depend on the studied zone. A typical matrix $F_{c c^{\prime}}$ is given, for zone 2 , by

$$
\left(\begin{array}{lll}
F_{w w} & F_{w v} & F_{w d} \\
F_{v w} & F_{v v} & F_{v d} \\
F_{d w} & F_{d v} & F_{d d}
\end{array}\right) \simeq\left(\begin{array}{ccc}
0.95 & 0.03 & 0.02 \\
0.58 & 0.38 & 0.04 \\
0.99 & 0.01 & 0.00
\end{array}\right)
$$

All the radiative energy which is emitted by the phases $w$ or $d$ and leaves the considered volume element of the system is practically absorbed by the interfaces $w$ of the adjacent volume elements. Indeed, the semitransparent phase is optically thin at the scale of the volume element. Moreover, the radiative energy emitted by the steam $v$ is mainly absorbed by the interfaces $w$ but also by the steam itself within the adjacent volume elements. The absorption efficiency of interfaces is due to the high value of $\mathrm{ZrO}_{2}$ averaged absorptivity, equal to 0.8 as shown in Fig.8.

\subsection{Contributions of the first order fluxes}

In a second step, the interfacial fluxes at the first order perturbation $\varphi_{w}^{(1)}$ have also been computed from Eqs. (33) and (34). The associated radiative Fourier law is only valid when the Gomart and Taine criterion given by Eq. (5) is fulfilled, i.e. when the temperature gradient fulfills the condition

$$
\left|\frac{\partial T_{w}}{\partial z}\right|<60 \mathrm{~K} / \mathrm{cm} \quad \text { at } 2000 \mathrm{~K}
$$

The criterion given by Eq. (40) allows radiative fluxes to be determined with a $10 \%$ accuracy.

Zeroth order exchanges between phases can then be compared with first orders ones. $\varphi_{w}^{(1)}$ practically depends only on the temperature gradient $\partial T_{w} / \partial z$. The perturbation technique is valid as long as $\varphi_{w}^{(1)}$ is small compared to $\varphi_{w}^{(0)}$. The critical value of 
$\partial T_{w} / \partial z$ such as $\varphi_{w}^{(1)} / \varphi_{w}^{(0)}=0.1$ is given by

$$
\left|\frac{\partial T_{w}}{\partial z}\right|^{c r}=\left|\frac{0.1 \mathcal{P}_{w}^{(0)}}{\frac{\partial}{\partial T_{w}}\left[\left(F_{w v}+F_{w d}\right) k_{z_{w}}-F_{v w} k_{z_{v}}\right]+\frac{\partial}{\partial T_{v}}\left[\left(F_{w v}+F_{w d}\right) k_{z_{w}}-F_{v w} k_{z_{v}}\right]}\right|^{1 / 2} .
$$

The critical axial temperature gradient defined by Eq. (41) has been computed for the 35 studied zones and for two couples of temperatures: i) $\left(T_{w}, T_{v}\right)=(2000 \mathrm{~K}, 600 \mathrm{~K})$, which corresponds to a strong non equilibrium observed when water is injected for cooling the reactor; ii) $\left(T_{w}, T_{v}\right)=(1500 \mathrm{~K}, 800 \mathrm{~K})$, observed when temperature of rods has decreased by heating surrounding steam. For all studied zones and for these two couples of temperatures, the first order exchanges are negligible as long as the temperature gradient does not exceed $300 \mathrm{~K} / \mathrm{cm}$. This condition is practically always verified, but is less restrictive than Eq. (40). Consequently, corrections associated with the first order perturbation are negligible for the considered application.

\section{Conclusion}

An original model has been developed for computing, after phase homogenization, coupled radiative transfer between the phases of a strongly anisotropic porous medium with opaque and semitransparent phases. The homogenized phase associated with opaque wall elements does not follow Beer's law related to radiation extinction. At the optically thick limit, effective extinction and scattering coefficients characterize this homogenized phase. They have been determined from the Generalized Radiative Transfer Equation developed by Taine et al. [1] for non Beerian porous media.

The radiative transfer model is based on a RTE with three source terms, which are associated with the temperature fields of the interfaces and of two semitransparent phases (steam and water droplets). This specific RTE has been solved by a perturbation technique, which allows radiative powers per unit volume and radiative interfacial fluxes, which are exchanged between phases at local scale, to be computed. Both contributions of zeroth order perturbation and corrections associated with the 
first order perturbation have been determined. In the second case, radiative fluxes have been expressed as coupled Fourier's laws which are characterized by radiative conductivity tensors associated with each phase.

This approach has been applied to a radiative model for a tentative case of reflooding a nuclear reactor core, in thermohydraulics conditions which correspond to some types of severe accident. The radiative statistical functions associated with the opaque interfaces of the small scale facility of a nuclear reactor core, which reproduces damages after an accident, have first been determined. These non Beerian statistical functions have been directly computed from $\gamma$-tomography images, by a Monte Carlo method. The radiative properties of the fluid phase, which is a mixture of steam and water droplets, have also been characterized. The orders of magnitude of the different contributions to the radiative interfacial flux have been compared. In this application, the first order perturbation contribution can be neglected.

The developed radiative model can be coupled, at local scale, with a model based on three local temperature fields, which would account for other heat transfer modes $[22]$.

\section{References}

[1] J. Taine, F. Bellet, V. Leroy, E. Iacona, Generalized radiative transfer equation for porous medium upscaling ; application to radiative fourier law, International Journal of Heat and Mass Transfer 53 (2010) 4071-4081.

[2] T. Hendricks, J. Howell, Inverse radiative analysis to determine spectral radiative properties using the discrete ordinates method, International Journal of Heat and Mass Transfer 2 (1994) 75-80. Brighton.

[3] T. Hendricks, J. Howell, Absorption/scattering coefficients and scattering phase function in reticulated porous ceramics, Journal of Heat Transfer 118 (1996) $79-87$.

[4] T. Hendricks, J. Howell, New radiative analysis approach for reticulated porous 
ceramics using discrete ordinates method, Journal of Heat Transfer 4 (1996) 911-917.

[5] D. Baillis, J. Sacadura, Identification of spectral radiative properties of polyurethane foam from hemispherical and bi-directional transmittance and reflectance measurement., Journal of Quantitative Spectroscopy and Radiative Transfer 73 (2002) 297-306.

[6] B. P. Singh, M. Kaviany, Modelling radiative heat transfer in packed beds, International Journal of Heat and Mass Transfer 35 (1992) 1397-1405.

[7] M. Loretz, R. Coquard, D. Baillis, D. Maire, Metallic foams : radiative properties; comparison between different models, Journal of Quantitative Spectroscopy and Radiative Transfer 109 (2008) 16-27.

[8] D. Baillis, J. Sacadura, Thermal radiation properties of dispersed media: theoretical prediction and experimental characterization, Journal of Quantitative Spectroscopy and Radiative Transfer 67 (2000) 327-363.

[9] W. Lipinski, J. Petrasch, S. Haussener, Application of the spatial averaging theorem to radiative heat transfer in two-phase media, Journal of Quantitative Spectroscopy and Radiative Transfer 11 (2010) 253-258.

[10] S. Whitaker, The Method of Volume Averaging, Kluwer Academics Publishers, 1999.

[11] M. Quintard, S. Whitaker, One- and two-equation models for transient diffusion processes in two-phase systems, Advances in Heat Transfer 23 (1993) 369-464.

[12] J. Consalvi, B. Porterie, J. Loraud, A formal averaging procedure for radiation heat transfer in particulate media, International Journal of Heat and Mass Transfer 45 (2002) 2755-2768.

[13] M. Tancrez, J. Taine, Direct identification of absorption and scattering coefficients and phase function of a porous medium by a Monte Carlo technique, International Journal of Heat and Mass Transfer 47 (2004) 373-383. 
[14] B. Zeghondy, E. Iacona, J. Taine, Determination of the anisotropic radiative properties of a porous material by radiative distribution function identification (RDFI)., International Journal of Heat and Mass Transfer 49 (2006) 2810-2819.

[15] B. Zeghondy, E. Iacona, J. Taine, Experimental validation of RDFI method predictions of statistically anisotropic porous medium radiative properties, International Journal of Heat and Mass Transfer 49 (2006) 3701-3707.

[16] J. Petrasch, P. Wyss, A. Steinfeld, Tomography-based Monte Carlo determination of radiative properties of reticulated porous ceramics, Journal of Quantitative Spectroscopy and Radiative Transfer 105 (2007) 180-197.

[17] S. Haussener, P. Coray, W. Lipinski, P. Wyss, A. Steinfeld, Tomography -based heat and mass transfer characterization of reticulate porous ceramics fot hightemperature processing, J. of Heat Transfer 131 (2009) 1-9.

[18] S. Haussener, W. Lipinski, P. Wyss, A. Steinfeld, Tomography -based analysis of radiative transfer in reacting packed beds undergoing a solid-gas thermochemical transformation, J. of Heat Transfer in press (2009).

[19] F. Bellet, E. Chalopin, F. Fichot, E. Iacona, , J. Taine, RDFI determination of anisotropic and scattering dependent radiative conductivity tensors in porous media: Application to rod bundles, International Journal of Heat and Mass Transfer 52 (2009) 1544-1551.

[20] M. Loretz, E. Maire, D. Baillis, Analytical modelling of the radiative properties of metalic foams: contribution of X-ray tomography, Advanced Engineering Materials 10 (2008) 352-360.

[21] H. Gomart, J. Taine, Validity criterion of the radiative fourier law for an absorbing and scattering medium, Phys. Rev. E 83 (2011) 021202.

[22] V. Leroy, B. Goyeau, J. Taine, Coupled upscaling approaches for convection and radiation in porous media, Transport in Porous Media (submitted 02/2012). 
[23] J. Taine, E. Iacona, Upscaling statistical methodology for radiative transfer in porous media: New trends, Journal of Heat Transfer 134 (2012) 031012.

[24] M. Born, E. Wolf, Principle of Optics, N.-Y: Pergamon Press, 7th edition, 1999.

[25] R. Siegel, J. Howell, Thermal Radiation Heat Transfert, Taylor and Francis, third edition, 1992.

[26] B. Berthet, J. Bonnin, S. Bayle, N. Hanniet, F. Jeury, S. Gaillot, Y. Garnier, C. Martin, M. Laurie, B. Siri, PHEBUS PF FPT1 - Preliminary Report, DOCUMENT PHEBUS PF IP/97/334, IPSN, 1997.

[27] J. Delesse, B. Le Saec, G. Vignoles, New data structure for the computation of equivalent properties in 3d porous media, in: K. Lee, D. C. Anderson (Eds.), Proceedings of 6th ACM symposium on Solid Modelling and Applications, ACM Press, N.Y., 2001, p. 301.

[28] A. Lacis, V. Oinas, A description of the correlated $k$ distribution method for modeling nongray gaseous absorption, thermal emission, and multiple scattering in vertically inhomogeneous atmospheres, Journal of Geophysical Research 96 (1991) 9027-9063.

[29] W. Malkmus, Random lorentz band model with exponential-tailed s-1 line intensity distribution function, Journal of Optical Society of America 57 (1967) 323-329.

[30] P. Rivière, A. Soufiani, J. Taine, An Approximate Data Base of $\mathrm{H}_{2} \mathrm{O}$ Infrared Lines for High Temperature Applications at Low Resolution. Statistical Narrowband Model Parameters, Journal of Quantitative Spectroscopy and Radiative Transfer 53 (1995) 221-234.

[31] P. Rivière, A. Soufiani, J. Taine, Correlated-k and fictitious gas method for $\mathrm{H}_{2} \mathrm{O}$ near $2.7 \mu \mathrm{m}$, Journal of Quantitative Spectroscopy and Radiative Transfer 48 (1992) 187-203. 
[32] C. Bohren, D. Hufmann, Absorption and scattering of light by small particles, N.-Y John Wiley, 1983.

[33] Y. Touloukian, D. DeWitt, Thermal radiative properties. Metallic elements and alloys, volume 7 of Thermophysical properties of matter, IFI/Plenum, 1970. 


\section{List of Figures}

1 Axial cross section of the intact system (above) and the degraded system (below) along the vertical $z$-axis (PHEBUS-FPT1, [26]). . . . 31

2 Example of image associated with a 2D cross-sectional $\gamma$-tomography; intact system (PHEBUS-FPT1, [26]) . . . . . . . . . . . . . 32

$3 \gamma$-tomographies of the zone 2 of PHEBUS-FPT1 and 14 zones of PHEBUS-FPT2, delimited axially by $\left(z_{\min }, z_{\max }\right)[\mathrm{mm}]$ and associated values of $\Pi$ and $A\left[m^{-1}\right] \ldots \ldots \ldots \ldots$

4 Extinction cumulative distribution function $G_{\text {ext }}\left(s^{\prime}-s, \theta=\pi / 2, \varphi\right)$ for $\theta=\pi / 2$ and different $\varphi$ values $\left(1.5^{\circ}+, 16.5^{\circ} \times, 31.5^{\circ} \circ, 43.5^{\circ}\right.$ $\square$ ) and corresponding extinction cumulative function over all $\varphi$ (thick

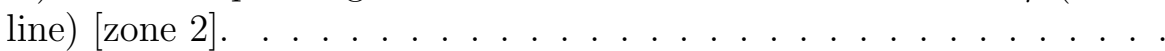

$5 \quad$ Directional generalized extinction coefficient at equilibrium $\left[\mathrm{mm}^{-1}\right]$ vs $\varphi$ for different values of $\theta\left(14.0^{\circ}+, 34.1^{\circ} \times, 44.9^{\circ} \circ, 53.9^{\circ} \square, 69.3^{\circ}\right.$ $\left.\triangle, 90^{\circ} \boldsymbol{\Delta}\right)$ [zone 2]. . . . . . . . . . . . . . . . . .

Directional scattering asymmetry parameter vs $\varphi$ for different values of $\theta\left(14.0^{\circ}+, 34.1^{\circ} \times, 44.9^{\circ} \circ, 53.9^{\circ} \square, 69.3^{\circ} \triangle, 90^{\circ} \mathbf{\Delta}\right)$ [zone 2]. .

7 Transmissivity of columns of lenghts $1 \mathrm{~cm}$ (a) and $5 \mathrm{~cm}$ (b) associated with interfaces (dashed line), water droplets at $\left(R_{d}=25 \mu \mathrm{m}, f_{v}=\right.$ $\left.10^{-3}\right)(\triangle)$, steam at temperature $T_{v}=1000 \mathrm{~K}$ and pressure $p_{v}=$ $1 \mathrm{~atm}(\times)$ or $p_{v}=5 \mathrm{~atm}(\mathrm{o}) \ldots \ldots \ldots \ldots \ldots$

8 Local absorptivity of Zirconium oxide $\mathrm{ZrO}_{2}$ vs $\mathrm{T}_{w}$ [33]. . . . . . . 38

9 Dimensionless radiative conductivities $k_{r}^{+}$et $k_{z}^{+}$vs $\Pi$ and corresponding fits. . . . . . . . . . . . . . . . . . . 39

$10-\mathcal{P}_{w}^{(0)}$ vs porosity associated with a semitransparent phase made of: i) Both steam and droplets of radius $R_{d}=50 \mu \mathrm{m}(\times)$ or $R_{d}=300 \mu \mathrm{m}$ (+); ii) Only steam (o); ii) Only droplets of radius $R_{d}=50 \mu \mathrm{m}(\square)$; $T_{w}=2000 \mathrm{~K}, T_{v}=600 \mathrm{~K}, f_{v}=10^{-3}$ and $p_{v}=5 \mathrm{~atm}$ (a) or $p_{v}=1 \mathrm{~atm}$ (b).

$\mathcal{P}_{v}^{(0)}$ vs porosity associated with a semitransparent phase made of: i) Both steam and droplets of radius $R_{d}=50 \mu \mathrm{m}(\times)$ or $R_{d}=300 \mu \mathrm{m}$ (+); ii) Only steam (o); $T_{w}=2000 K, T_{v}=600 K, f_{v}=10^{-3}$ and

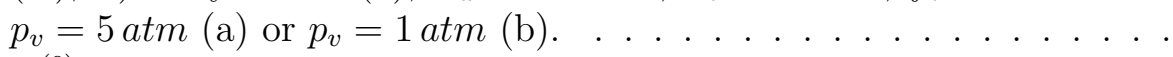

$12 \mathcal{P}_{d}^{(0)}$ vs porosity associated with a semitransparent phase made of: i) Both steam and droplets of radius $R_{d}=50 \mu \mathrm{m}(\times)$ or $R_{d}=300 \mu \mathrm{m}$ (+); ii) Only droplets of radius $R_{d}=50 \mu \mathrm{m}(\square) ; T_{w}=2000 \mathrm{~K}, T_{v}=$ $600 \mathrm{~K}, f_{v}=10^{-3}$ and $p_{v}=5 \mathrm{~atm}$ (a) or $p_{v}=1 \mathrm{~atm}(\mathrm{~b}) \ldots \ldots$. . 
13 Dimensionless quantities $F_{w w} k_{r_{w}} / k_{r}^{\text {trans }}$ (a) and $F_{w w} k_{z_{w}} / k_{z}^{\text {trans }}$ (b) vs porosity associated with a semitransparent phase made of: i)Both steam and droplets of radius $R_{d}=50 \mu \mathrm{m}(\times)$ or $R_{d}=300 \mu \mathrm{m}(+)$; ii) Only steam (o); iii) Only droplets of radius $R_{d}=50 \mu \mathrm{m}(\square) ; T_{w}=$ $2000 \mathrm{~K}, T_{v}=600 \mathrm{~K}, f_{v}=10^{-3}, p_{v}=5 \mathrm{~atm} \ldots \ldots \ldots$ 

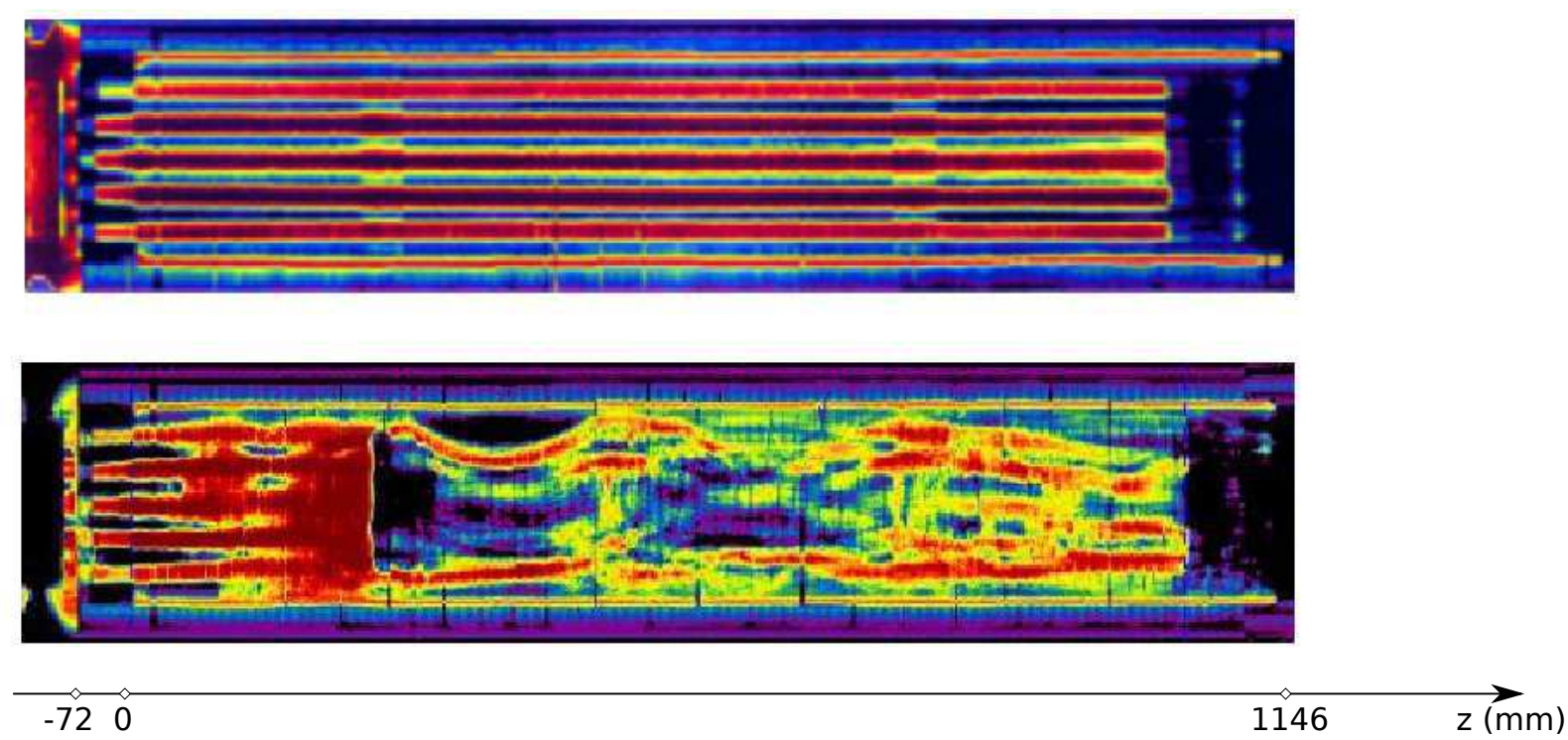

Figure 1: Axial cross section of the intact system (above) and the degraded system (below) along the vertical $z$-axis (PHEBUS-FPT1, [26]). 


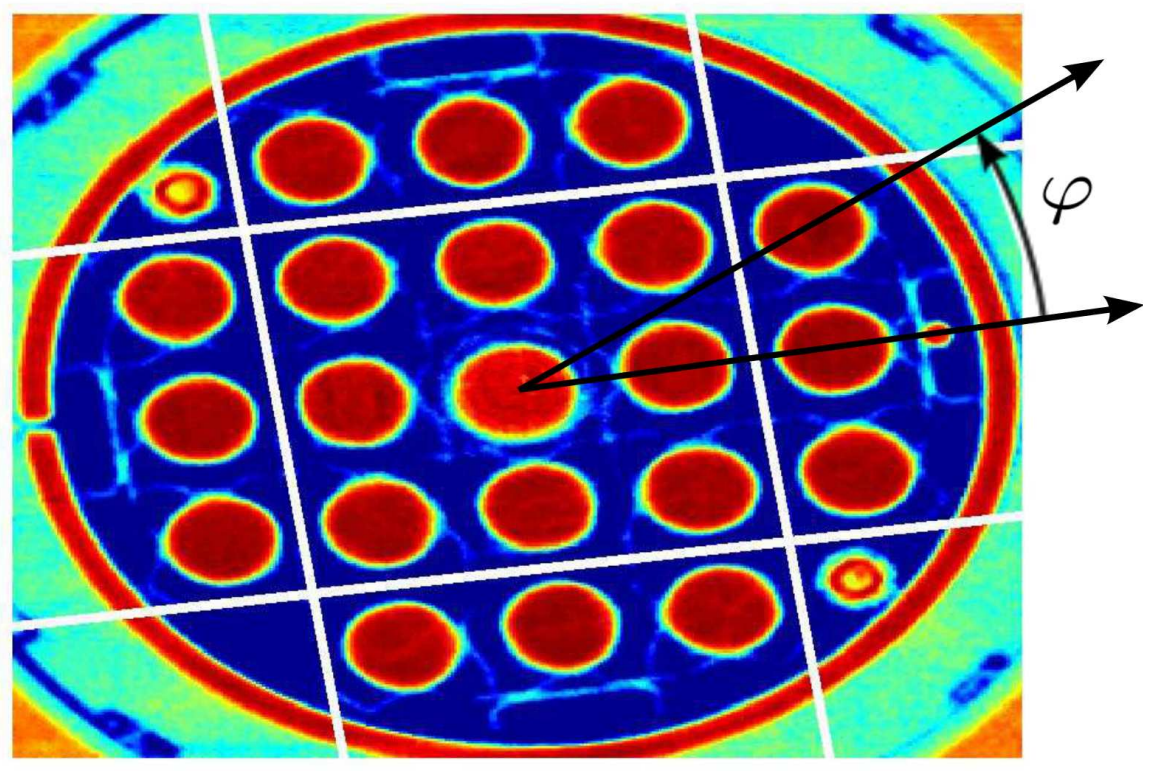

Figure 2: Example of image associated with a 2D cross-sectional $\gamma$-tomography; intact system (PHEBUS-FPT1, [26]). 


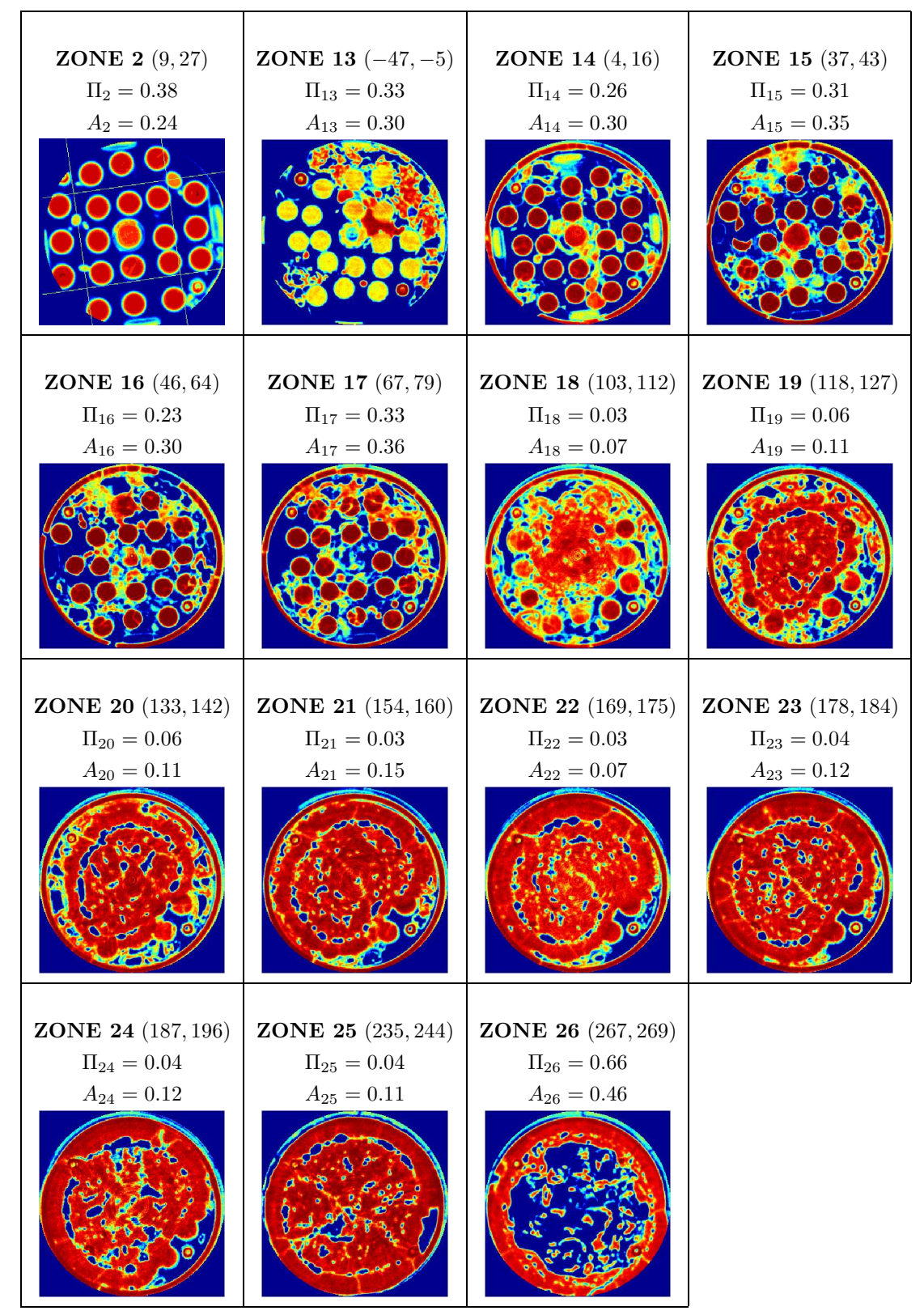

Figure 3: $\gamma$-tomographies of the zone 2 of PHEBUS-FPT1 and 14 zones of PHEBUSFPT2, delimited axially by $\left(z_{\min }, z_{\max }\right)[\mathrm{mm}]$ and associated values of $\Pi$ and $A$ $\left[\mathrm{mm}^{-1}\right]$. 


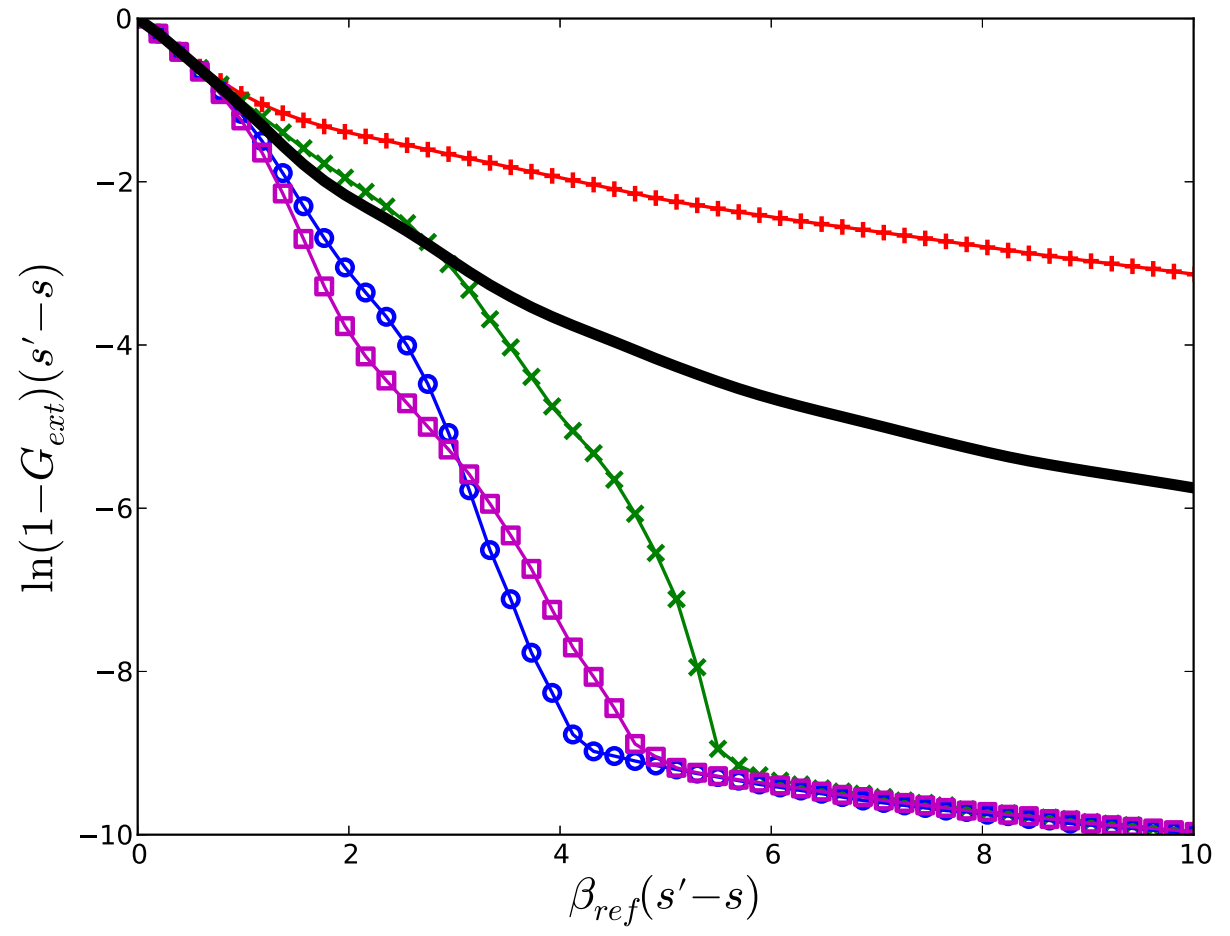

Figure 4: Extinction cumulative distribution function $G_{\text {ext }}\left(s^{\prime}-s, \theta=\pi / 2, \varphi\right)$ for $\theta=\pi / 2$ and different $\varphi$ values $\left(1.5^{\circ}+, 16.5^{\circ} \times, 31.5^{\circ} \circ, 43.5^{\circ} \square\right)$ and corresponding extinction cumulative function over all $\varphi$ (thick line) [zone 2]. 


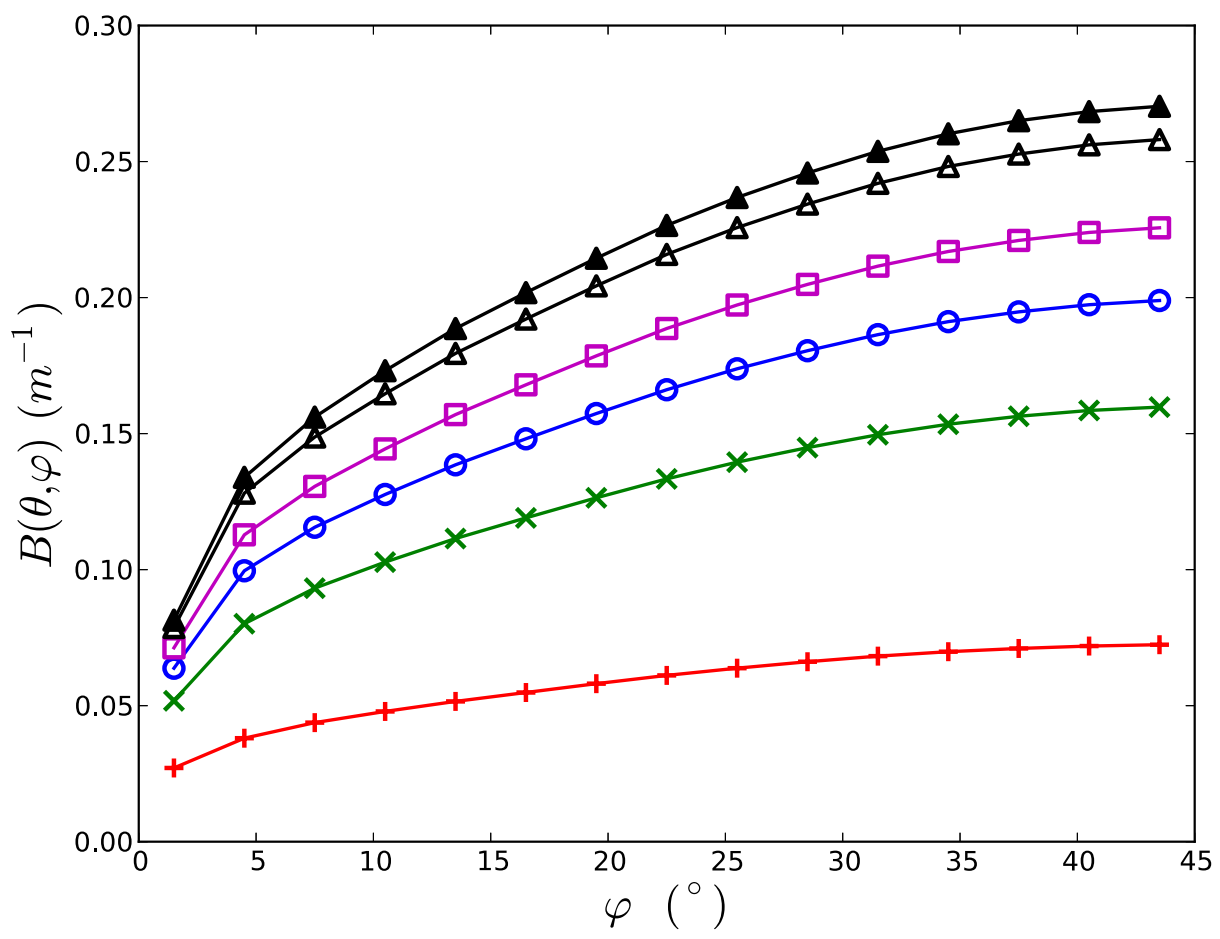

Figure 5: Directional generalized extinction coefficient at equilibrium $\left[\mathrm{mm}^{-1}\right]$ vs $\varphi$ for different values of $\theta\left(14.0^{\circ}+, 34.1^{\circ} \times, 44.9^{\circ} \circ, 53.9^{\circ} \square, 69.3^{\circ} \triangle, 90^{\circ} \mathbf{\Delta}\right)$ [zone 2]. 


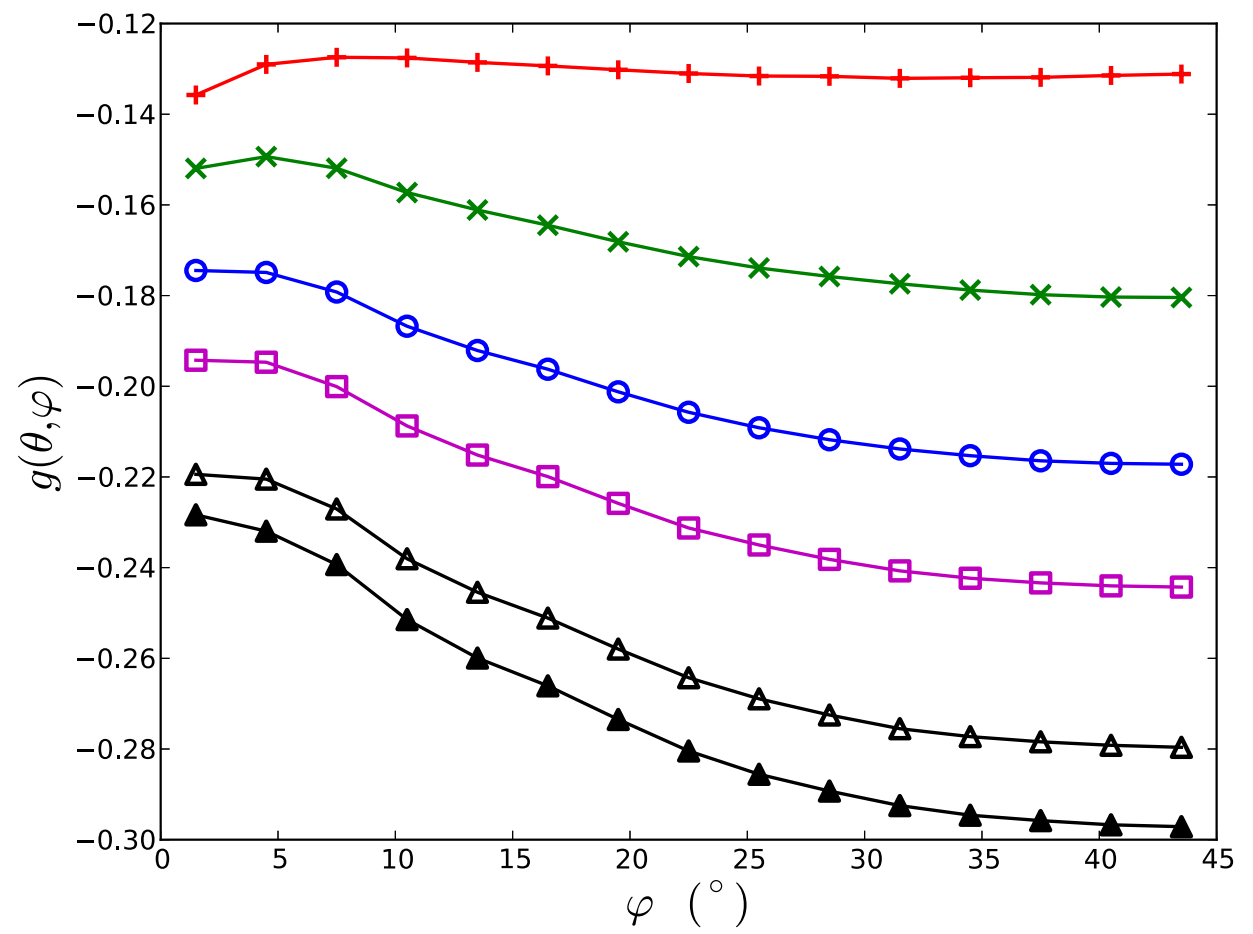

Figure 6: Directional scattering asymmetry parameter vs $\varphi$ for different values of $\theta$ $\left(14.0^{\circ}+, 34.1^{\circ} \times, 44.9^{\circ} \circ, 53.9^{\circ} \square, 69.3^{\circ} \triangle, 90^{\circ} \mathbf{\Delta}\right)$ [zone 2]. 

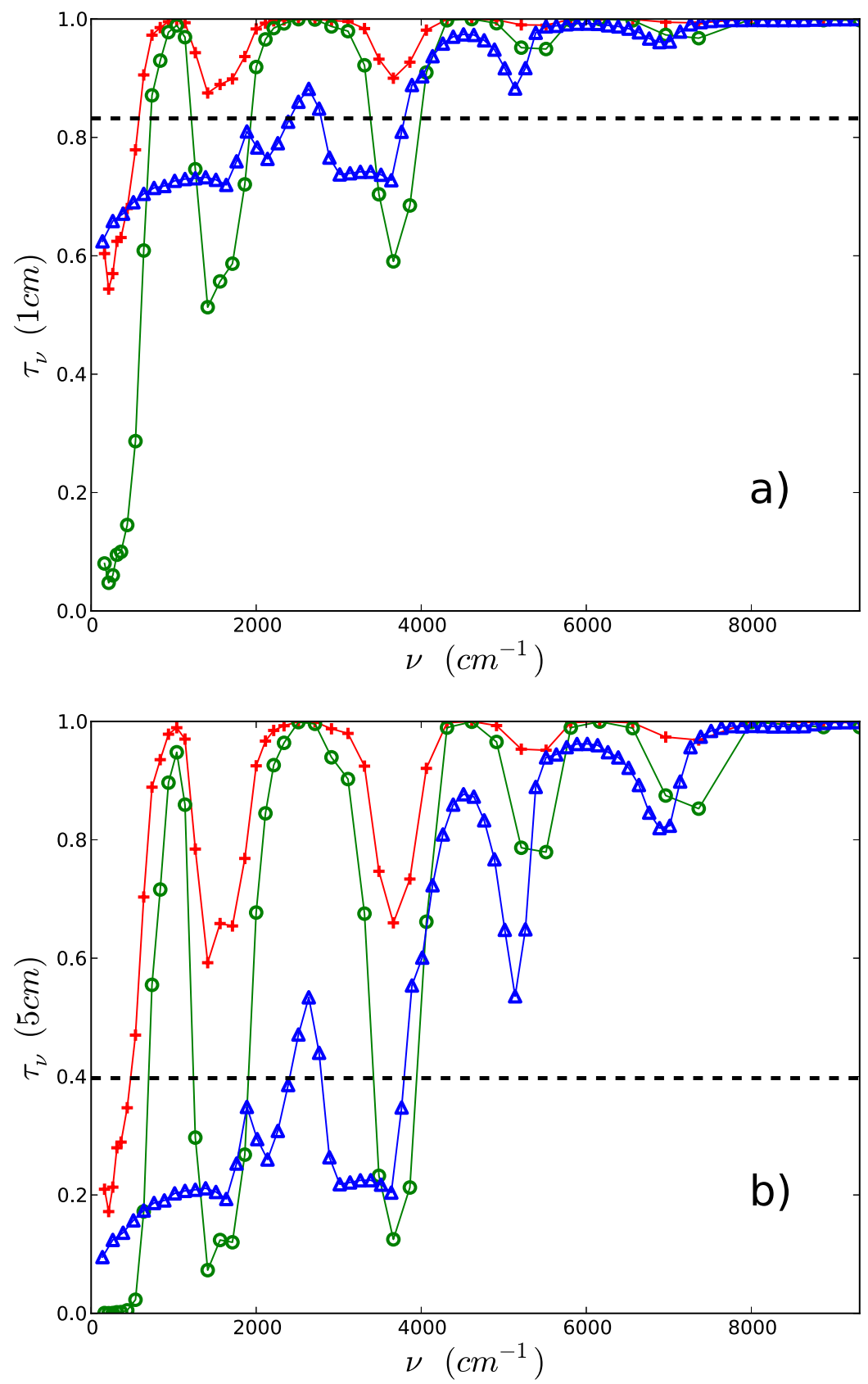

Figure 7: Transmissivity of columns of lenghts $1 \mathrm{~cm}$ (a) and $5 \mathrm{~cm}$ (b) associated with interfaces (dashed line), water droplets at $\left(R_{d}=25 \mu \mathrm{m}, f_{v}=10^{-3}\right)(\triangle)$, steam at temperature $T_{v}=1000 \mathrm{~K}$ and pressure $p_{v}=1 \mathrm{~atm}(\times)$ or $p_{v}=5 \mathrm{~atm}(\mathrm{\circ})$. 


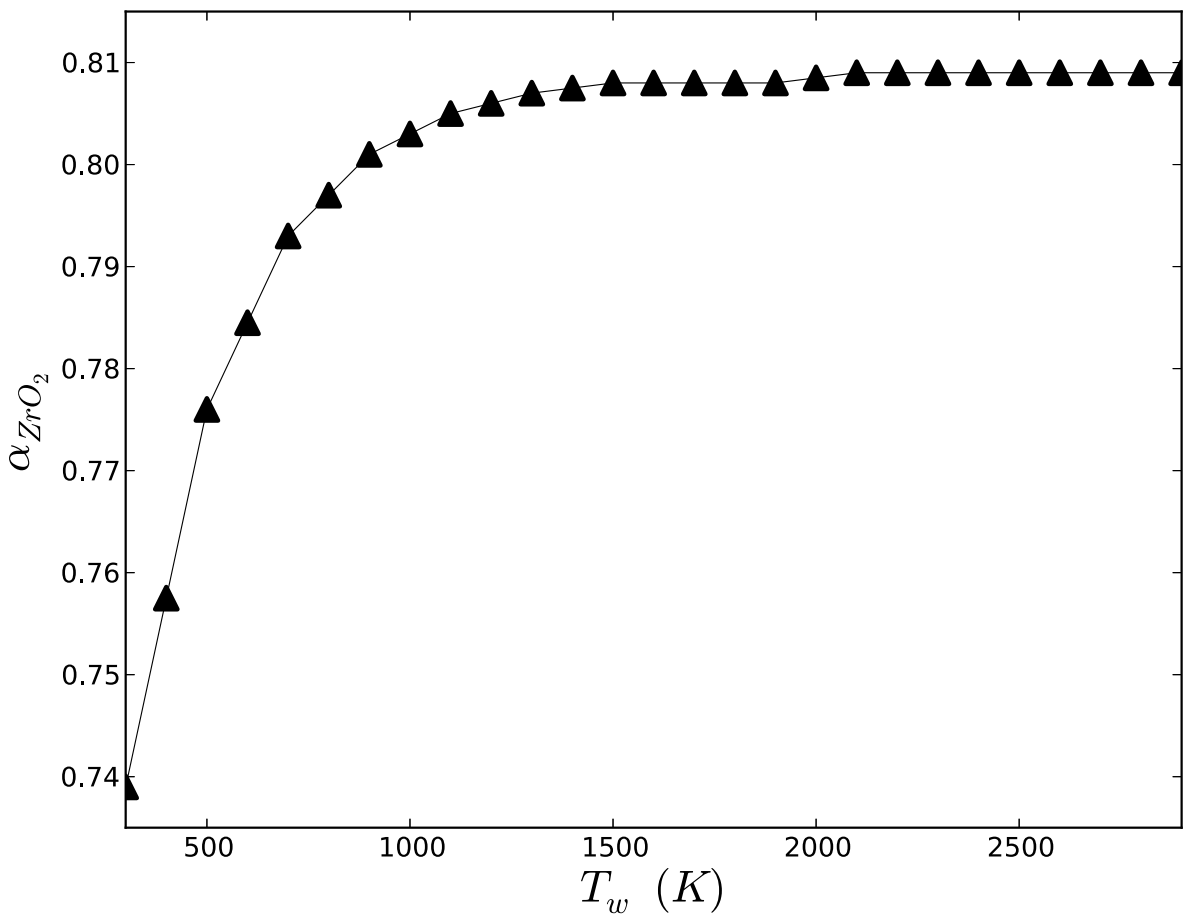

Figure 8: Local absorptivity of Zirconium oxide $\mathrm{ZrO}_{2}$ vs $T_{w}$ [33]. 


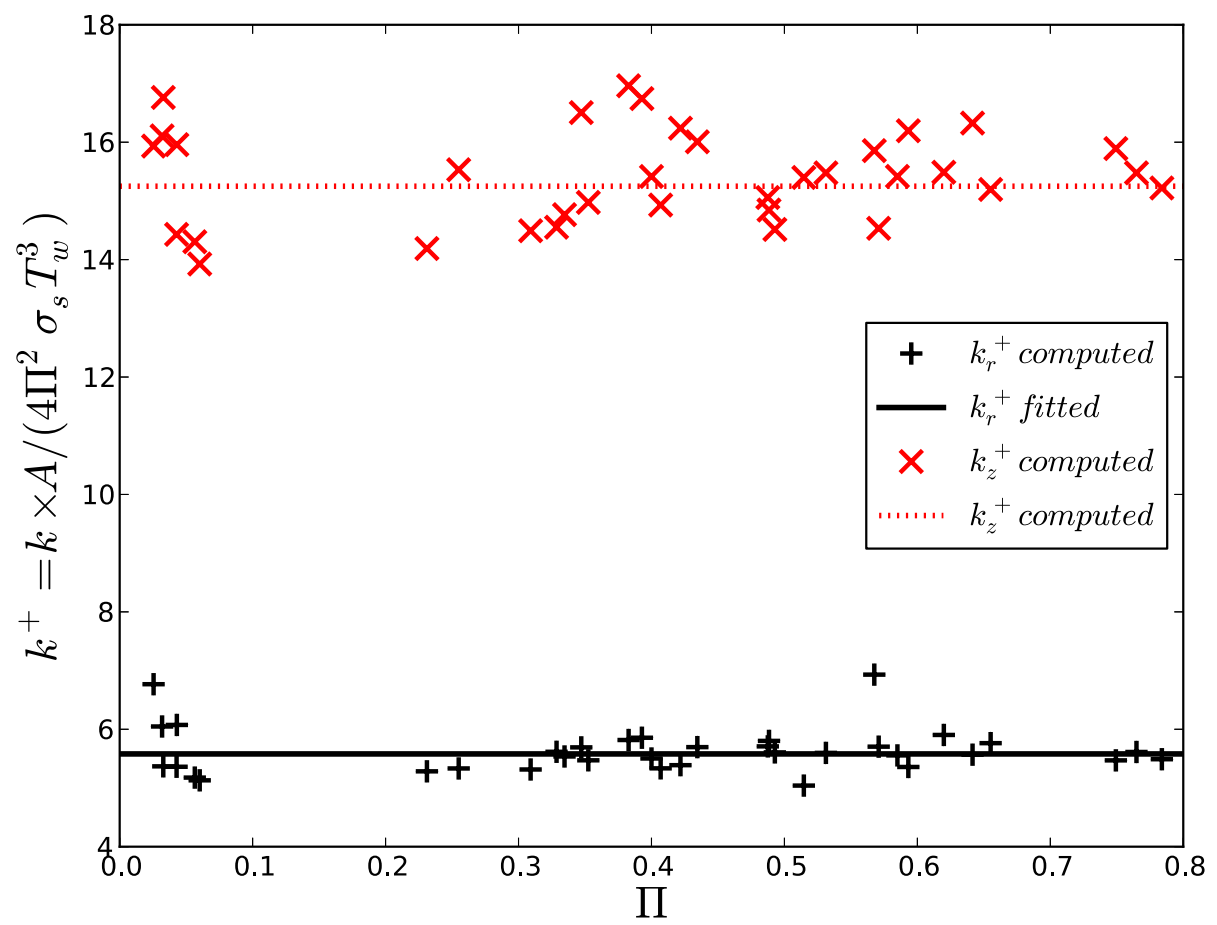

Figure 9: Dimensionless radiative conductivities $k_{r}^{+}$et $k_{z}^{+}$vs $\Pi$ and corresponding fits. 

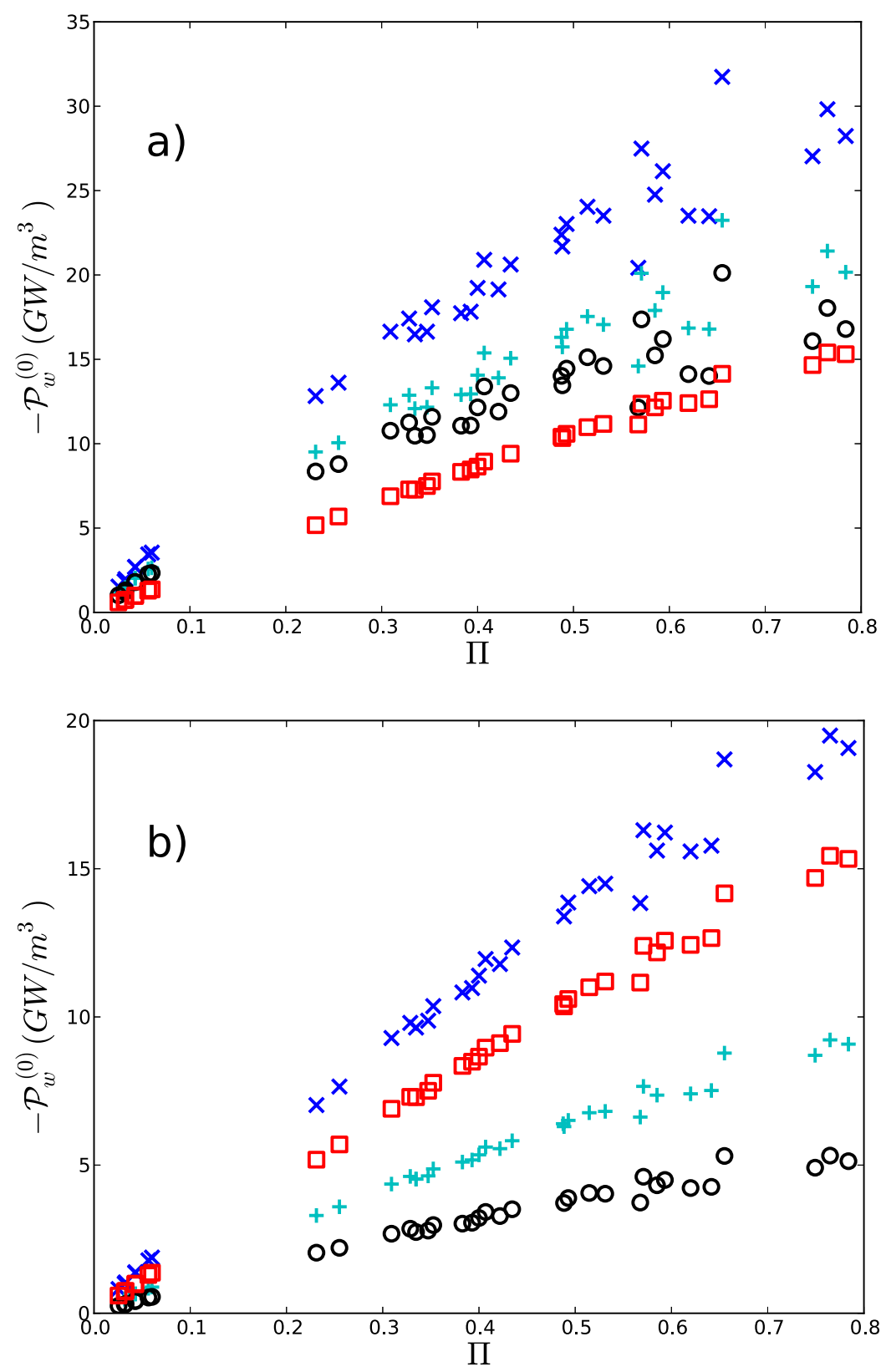

Figure 10: $\quad-\mathcal{P}_{w}^{(0)}$ vs porosity associated with a semitransparent phase made of: i) Both steam and droplets of radius $R_{d}=50 \mu \mathrm{m}(\times)$ or $R_{d}=300 \mu \mathrm{m}(+)$; ii) Only steam (o); ii) Only droplets of radius $R_{d}=50 \mu \mathrm{m}(\square) ; T_{w}=2000 \mathrm{~K}, T_{v}=600 \mathrm{~K}$, $f_{v}=10^{-3}$ and $p_{v}=5$ atm (a) or $p_{v}=1$ atm (b). 

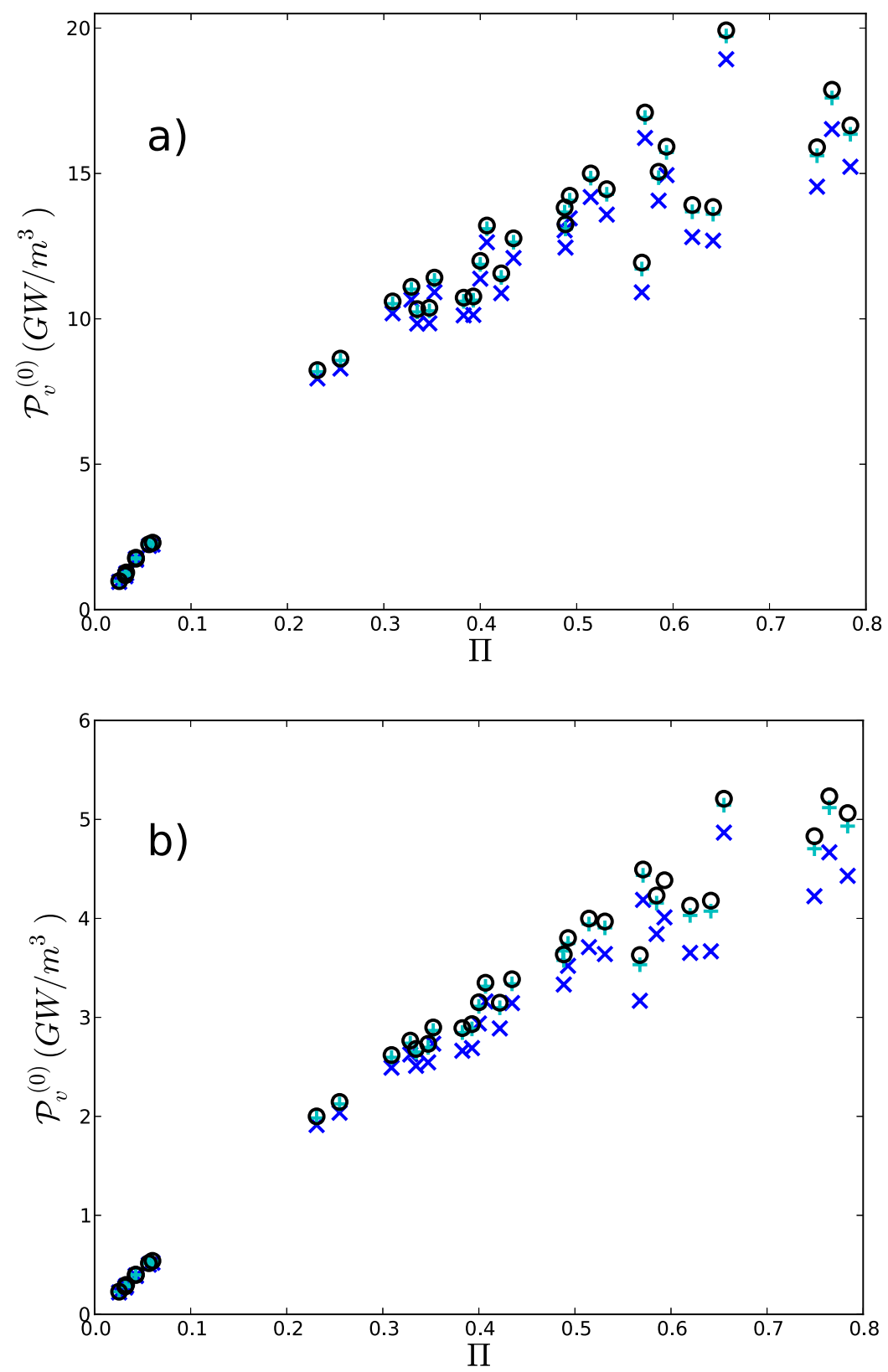

Figure 11: $\mathcal{P}_{v}^{(0)}$ vs porosity associated with a semitransparent phase made of: i) Both steam and droplets of radius $R_{d}=50 \mu \mathrm{m}(\times)$ or $R_{d}=300 \mu \mathrm{m}(+)$; ii) Only steam (o); $T_{w}=2000 \mathrm{~K}, T_{v}=600 \mathrm{~K}, f_{v}=10^{-3}$ and $p_{v}=5 \mathrm{~atm}$ (a) or $p_{v}=1 \mathrm{~atm}$ (b). 

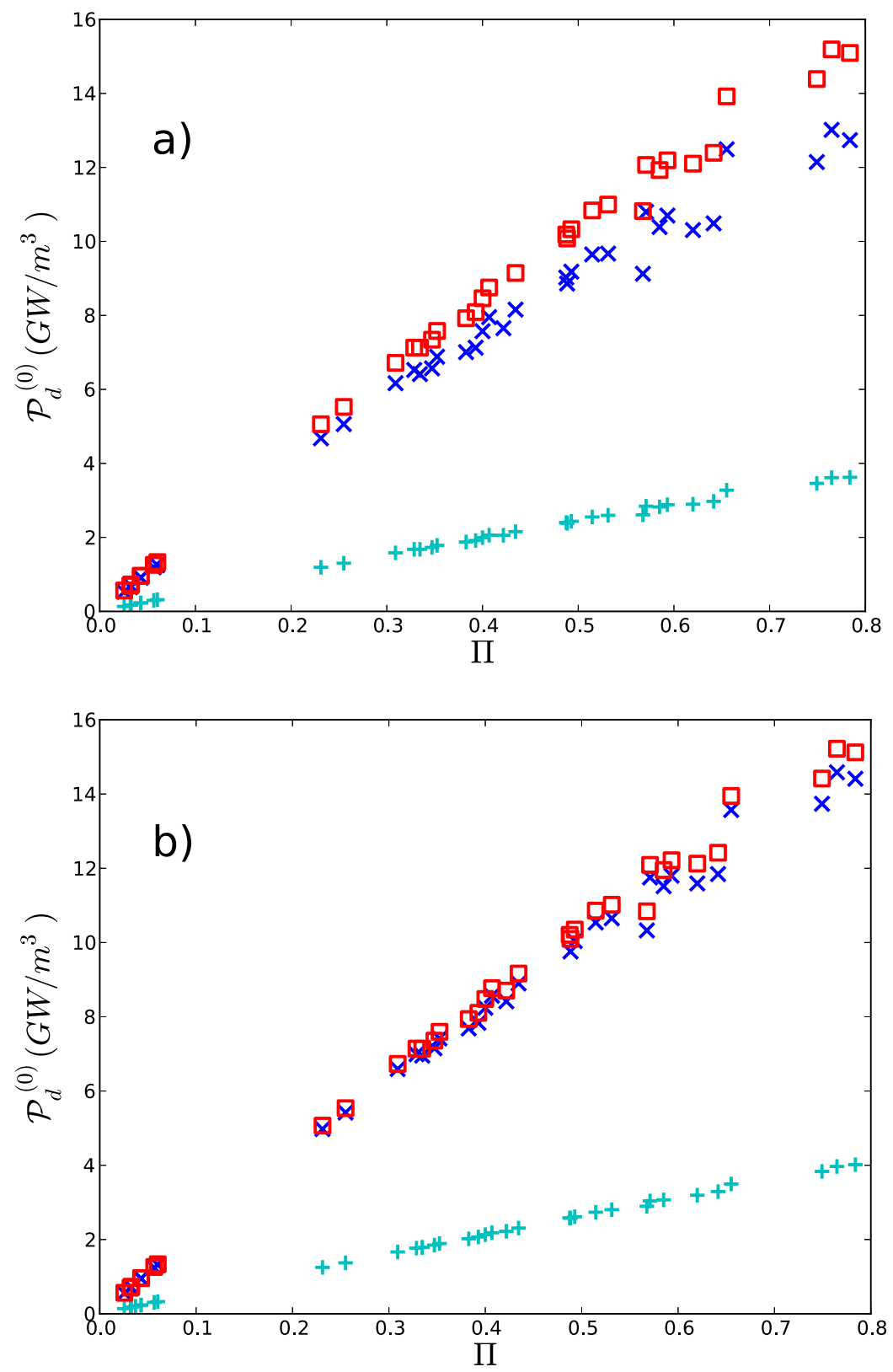

Figure 12: $\mathcal{P}_{d}^{(0)}$ vs porosity associated with a semitransparent phase made of: i) Both steam and droplets of radius $R_{d}=50 \mu \mathrm{m}(\times)$ or $R_{d}=300 \mu \mathrm{m}(+)$; ii) Only droplets of radius $R_{d}=50 \mu \mathrm{m}(\square) ; T_{w}=2000 \mathrm{~K}, T_{v}=600 \mathrm{~K}, f_{v}=10^{-3}$ and $p_{v}=5 \mathrm{~atm}$ (a) or $p_{v}=1 \mathrm{~atm}(\mathrm{~b})$. 

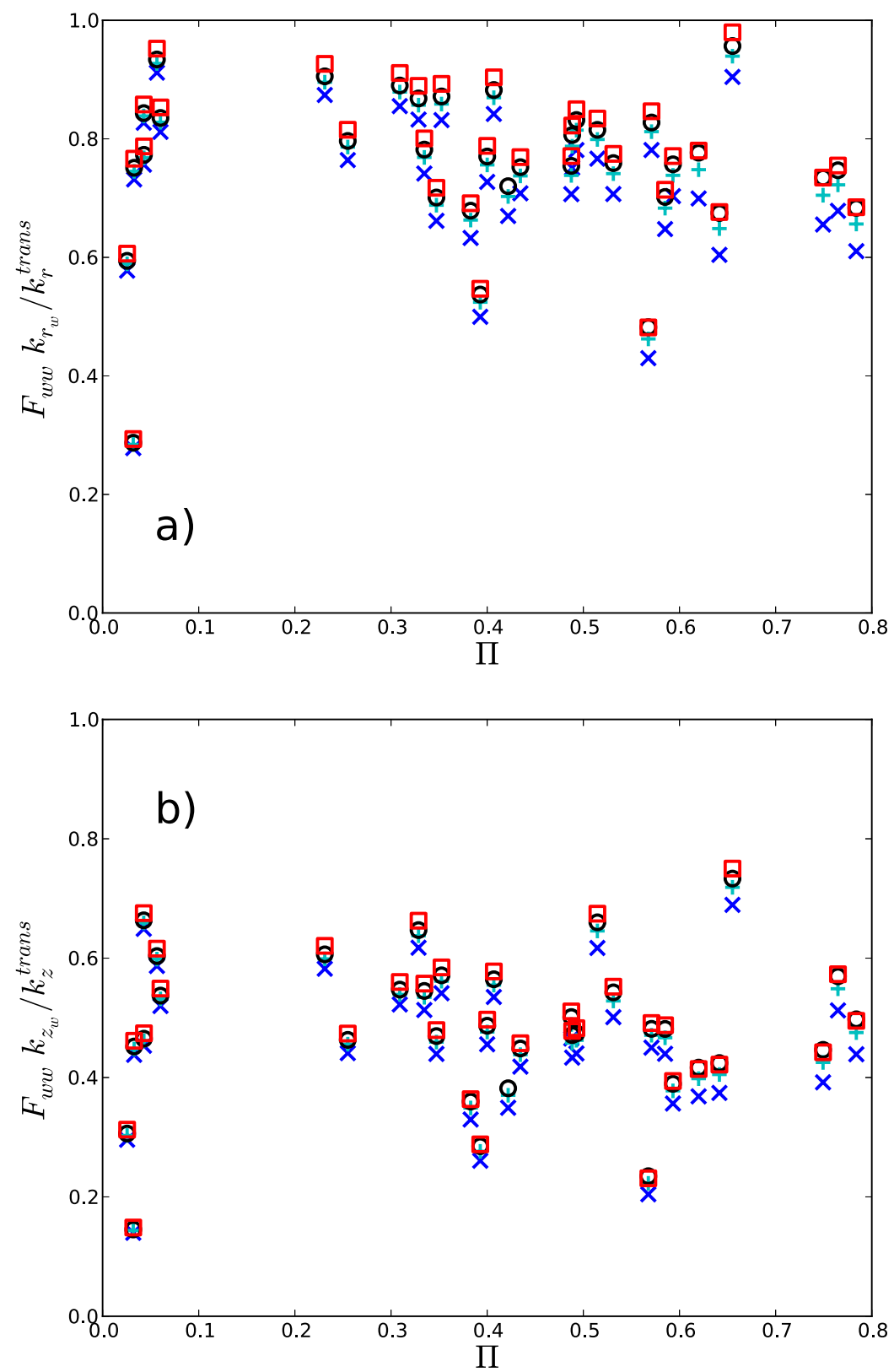

Figure 13: Dimensionless quantities $F_{w w} k_{r_{w}} / k_{r}^{\text {trans }}$ (a) and $F_{w w} k_{z_{w}} / k_{z}^{\text {trans }}$ (b) vs porosity associated with a semitransparent phase made of: i)Both steam and droplets of radius $R_{d}=50 \mu \mathrm{m}(\times)$ or $R_{d}=300 \mu \mathrm{m}(+)$; ii) Only steam (o); iii) Only droplets of radius $R_{d}=50 \mu \mathrm{m}(\square) ; T_{w}=2000 \mathrm{~K}, T_{v}=600 \mathrm{~K}, f_{v}=10^{-3}, p_{v}=5 \mathrm{~atm}$. 


\section{List of Tables}

1 Computed fluxes for the different types of fluid phase. . . . . . . . . 45 


\begin{tabular}{|c|c|}
\hline Fluid phase & Computed values \\
\hline \hline & $k_{r}^{\text {trans }}, k_{z}^{\text {trans }}$ \\
Transparent & $\varphi_{w}^{(0)}, \mathcal{P}_{v}^{(0)}$ \\
\hline Water steam at & $k_{r_{w}}, k_{z_{w}}$ \\
$1 \leq p_{v} \leq 10$ atm & $k_{r_{v}}, k_{z_{v}}$ \\
$T_{\text {sat }}\left(p_{v}\right) \leq T_{v} \leq 2900 K$ & $F_{w w}, F_{w v}$ \\
& $F_{v w}, F_{v v}$ \\
& \\
Water droplets at $T_{d}=373 K$ & $\varphi_{w}^{(0)}, \mathcal{P}_{d}^{(0)}$ \\
$f_{v}=10^{-3}$ & $k_{r_{w}}, k_{z_{w}}$ \\
$25 \mu m \leq R_{d} \leq 1$ mm & $F_{w w}, F_{w d}$ \\
& $F_{d w}, F_{d d}$ \\
& \\
Water steam and droplets at & $\varphi_{w}^{(0)}, \mathcal{P}_{v}^{(0)}, \mathcal{P}_{d}^{(0)}$ \\
$1 \leq p_{v} \leq 10$ atm & $k_{r_{w}}, k_{z_{w}}$ \\
$T_{d}=T_{\text {sat }}\left(p_{v}\right) \leq T_{v} \leq 2900 K$ & $k_{r_{v}}, k_{z_{v}}$ \\
$f_{v}=10^{-3}$ & $F_{w w}, F_{w v}, F_{w d}$ \\
$25 \mu m \leq R_{d} \leq 1$ mm & $F_{v w}, F_{v v}, F_{v d}$ \\
& $F_{d w}, F_{d v}, F_{d d}$ \\
\hline
\end{tabular}

Table 1: Computed fluxes for the different types of fluid phase. 